\title{
THE APSIDES OF GENERAL DYNAMICAL SYSTEMS*
}

BY

J. L. SYNGE

\section{PART I. INTRODUCTORY}

1. Introduction. In 1897 Hadamard $\dagger$ developed results concerning the motion of a general dynamical system, based on an examination of the sign of $d^{2} S / d t^{2}$, where $S$ is a function of the coördinates of the system. By using the methods of tensor calculus, it is possible to obtain the basis of Hadamard's results in a very compact way; this is done in the present paper, which then proceeds to develop further general results by the application of the method.

The paper consists of three Parts. In Part I, the word apse (borrowed from the theory of central orbits) is given a general definition and general apsidal properties are deduced from the equations of motion.

Part II deals with radial-apsides, which occur when the geodesic distance of the system from a fixed configuration has a stationary value, the geodesic distance being measured in the manifold of configurations with the lineelement defined in equation (5). The particular cases where the manifold is flat or radially symmetrical are discussed in $\$ 6$; a flat manifold with homogeneous potential energy is treated in $\$ 7$. For a general manifold, radialapsides near a position of instantaneous rest ( $\$ 8)$, near a position of equilibrium $(\$ 9)$, and near a pole of the potential energy $(\$ 10)$ are discussed.

Part III deals with potential-apsides. The case of a flat manifold with homogeneous potential energy is treated in $\$ 12$. For a general manifold, potential-apsides near a surface of instantaneous rest $(\$ 13)$ and near a position of equilibrium $(\$ 14)$ are dealt with. The theory of potential-apsides appears to be less simple than that of radial-apsides.

2. The apsides defined. Let there be a dynamical system with $N$ coördinates $x^{i}$. Let $S$ be any function of these coördinates. We define an $S$-apse on a trajectory to be a configuration where

$$
\frac{d S}{d t}=0 \text {. }
$$

The function $S$ will generally be either a maximum or a minimum at an $S$-apse, and we may distinguish the apsides as follows:

* Presented to the Society, December 28, 1931; received by the editors January 12, 1932.

† Sur certaines propriétés des trajectoires en dynamique, Journal de Mathématiques, (5), vol. 3 (1897), pp. 331-387. 


$$
\begin{aligned}
& \text { minimum } S \text {-apse : } \frac{d^{2} S}{d t^{2}}>0 ; \\
& \text { maximum } S \text {-apse }: \frac{d^{2} S}{d t^{2}}<0 .
\end{aligned}
$$

Let us view the apsides geometrically, taking as our space the $N$-dimensional manifold of configurations or $x$-space. We shall refer to a sub-space of $N-1$ dimensions as a surface. The equations

$$
S=\text { const. }
$$

define a family of surfaces; each surface has a positive side and a negative side, the positive side of the surface $S=C$ being that for which $S>C$. An $S$-apse is a point at which a trajectory touches one of the surfaces (3), or where the system is instantaneously at rest. In the latter case the apse is a point of reversal on the trajectory.

We are of course at liberty to choose the function $S$ as we please, but there are two special choices which claim attention. We shall for simplicity confine ourselves to holonomic dynamical systems which possess a potential energy and have no moving constraints (conservative statonomic holonomic systems). Let $V$ be the potential energy and

$$
T=\frac{1}{2} a_{i j} \dot{x}^{i} \dot{x}^{j}
$$

the kinetic energy, the usual summation convention being employed. In the geometry of the space, we shall employ the kinematical line-element given by

$$
d s^{2}=2 T d t^{2}=a_{i j} d x^{i} d x^{j} .
$$

We shall define a potential-apse or $V$-apse by (1) with $V$ substituted for $S$. The potential-apsides on a trajectory are therefore those points where the trajectory touches an equipotential surface or where it meets the surface $V=E$ ( $E$ being the constant total energy for the trajectory), since the system then necessarily comes to rest.

Let $O$ be any fixed point of the $x$-space, and $P$ a variable point. Let us write

$$
r=O P, F=\frac{1}{2} r^{2}
$$

$O P$ being measured along the geodesic joining these points. We shall define a radial-apse or $F$-apse by (1) with $F$ substituted for $S$. The radial-apsides on a trajectory are therefore those points where the trajectory touches one of the spheres $F=$ const. or where it meets the surface $V=E$; if the trajectory passes through $O$, we have also (in terms of the definition) a radial-apse there pro- 
vided that $d r / d t$ does not become infinite; such a point may be regarded as a point of contact with an infinitesimal sphere.

As we have seen, the apsidal properties of a trajectory refer generally to the contacts of the trajectory with the surfaces (3), which, in the two special cases considered, are respectively the equipotential surfaces and the spheres having $O$ for center. Certain familiar dynamical systems possess simple apsidal properties, notably, a particle in a central field of force depending only on the distance, the spherical pendulum, and the symmetrical top. In the classical theory of central orbits, the equipotential surfaces coincide with the spheres, and the two types of apse are no longer distinct. The same is true for the spherical pendulum, when treated geometrically with the metric (5), the point $O$ being one of the two positions of equilibrium of the pendulum, the highest and lowest points of the sphere. The case of the symmetrical top is not quite so simple; here each equipotential surface may be described as a "circular cylinder," being the envelope of a singly infinite family of spheres of constant radius having their centers on one of two certain closed geodesics composed of all the configurations in which the axis of the top is directed vertically upwards, or downwards, respectively. Every potential-apse is therefore a radial-apse for some suitably chosen center $O$ on one of the closed geodesics. The systems in question owe their simple apsidal properties to the simple symmetries which they enjoy.

3. The equations of motion. As regards notation, we shall denote covariant differentiation with respect to the fundamental tensor $a_{i j}$ merely by the addition of a subscript. The absolute derivative of a vector $X^{i}$, defined along a curve $x^{i}=x^{i}(u)$, where $u$ is any parameter, will be written

$$
\frac{\delta X^{i}}{\delta u}=\frac{d X^{i}}{d u}+\left\{\begin{array}{c}
i \\
j k
\end{array}\right\} X^{j} \frac{d x^{k}}{d u} .
$$

If $v^{i}$ denotes the velocity $\left(v^{i}=d x^{i} / d t\right)$, the equations of motion are*

$$
\frac{\delta v^{i}}{\delta t}=-V^{i}
$$

The system possesses the integrảl of energy

$$
v^{2}=2(E-V)
$$

where

$$
v^{2}=v_{i} v^{i}=2 T
$$

* On the geometry of dynamics, Philosophical Transactions, Royal Society, (A), vol. 226 (1926), pp. 31-106. The notation has been altered. 
If $S$ is any function of position, we have

or, by (8) and (9),*

$$
\begin{aligned}
\frac{d S}{d t} & =S_{i} v^{i}, \\
\frac{d^{2} S}{d t^{2}} & =S_{i} \frac{\delta v^{i}}{\delta t}+S_{i j} v^{i} v^{j},
\end{aligned}
$$

$$
\frac{d^{2} S}{d t^{2}}=-S_{i} V^{i}+2(E-V) S_{i j} \lambda^{i} \lambda^{i},
$$

where $\lambda^{i}$ is the unit vector tangent to the trajectory, defined by

$$
\lambda^{i}=v^{i} / v .
$$

4. The general apsidal properties. Let us write

$$
\psi_{S}(x, \lambda, E)=2(E-V) S_{i j} \lambda^{i} \lambda^{j}-S_{i} V^{i},
$$

the notation indicating that it is a function of position, of direction, and of the total energy, calculated with respect to the function $S$. We shall call $\psi_{s}$ the $S$-apsidal function.

By (13), we have

$$
\frac{d^{2} S}{d t^{2}}=\psi_{S}(k, \lambda, E) .
$$

The investigations of Hadamard are based on the following elementary facts:
(A) If at $t=t_{1}$
and if for
we have
$t_{1} \leqq t \leqq t_{2}$
then during
we have

$$
t_{1} \leqq t \leqq t_{2}
$$

(i) $d S / d t>0$,

$$
\psi_{s}>0
$$

$S$ increases steadily;

(ii) $d S / d t<0$, $\psi_{s}>0$, there is at most one $S$-apse,

(iii) $d S / d t>0$,

$$
\psi_{s}<0 \text {, }
$$
and it is a minimum apse;

(iv) $d S / d t<0$,

$\psi_{s}<0$, there is at most one $S$-apse, and it is a maximum apse;

(B) Between two $S$-apsides, there must be at least one zero of the $S$-apsidal function $\psi_{s}$.

(C) If at every point of a region $R, \psi_{s}$ is positive [negative] for all directions $\lambda^{i}$ tangent to the surface $S=$ const. through the point, then the only apsides possible in $R$ are minimum [maximum] apsides.

* Cf. Hadamard, loc. cit., p. 361. 
Hadamard* has given (without proof) geometrical interpretations of the part of $\psi_{S}$ which we have written $S_{i j} \lambda^{i} \lambda^{i}$. These interpretations are immediate when the tensor calculus is employed. If we draw a geodesic of the $x$-space in the direction $\lambda^{i}$ at a point $P$, and if $\mu^{i}$ is the unit tangent vector of this geodesic at any point, then

$$
\frac{d S}{d s_{\vartheta}}=S_{i} \mu^{i}, \frac{d^{2} S}{d s_{o}{ }^{2}}=S_{i j \mu^{i} \mu^{i}}
$$

so that

$$
\left(\frac{d^{2} S}{d s_{\vartheta}^{2}}\right)_{P}=S_{i j} \lambda^{i} \lambda^{i}
$$

$d s_{\text {o }}$ being an element of the arc of the geodesic.

Now let $\lambda^{i}$ at $P$ be tangent to the surface $S=$ const. through $P$, so that

$$
S_{i} \lambda^{i}=0
$$

at the point. Let us draw a geodesic of $S=$ const. having the initial direction indicated by $\lambda^{i}$, and let $\mu^{i}$ be the unit tangent vector of this geodesic. Then, using the first Frenet formula,

$$
S_{i} \mu^{i}=0, \quad S_{i j} \mu^{i} \mu^{j}+\kappa S_{i} \stackrel{i}{i}(1)=0,
$$

along the geodesic, $\kappa$ being its first curvature $(\kappa>0)$ and $\mu_{(1)}^{i}$ its unit first normal vector. Let $n^{i}$ be the unit normal to $S=$ const., pointing to the positive side of the surface, so that

$$
n^{i}=S^{i} /\left(S_{j} S^{i}\right)^{1 / 2},
$$

and let $\epsilon= \pm 1$ according as $\mu_{(1)}^{i}$ points to the positive or negative side of $S=$ const., so that

$$
\stackrel{i}{\mu_{(1)}}=\epsilon n^{i} .
$$

Then, taking the second of (20) at the point $P$ (where $\mu^{i}=\lambda^{i}$ ) we have

$$
S_{i j} \lambda^{i} \lambda^{i}=-\epsilon \kappa\left(S_{i} S^{i}\right)^{1 / 2} .
$$

We may define the curvature of $S=$ const. for the direction $\lambda^{i}$ to be

$$
k(S, \lambda)=-\epsilon \kappa,
$$

so that the curvature is positive when the surface is convex on its positive side; then

$$
S_{i j} \lambda^{i} \lambda^{i}=k(S, \lambda)\left(S_{i} S^{i}\right)^{1 / 2},
$$

\footnotetext{
* Loc. cit., p. 362.
} 
for any direction $\lambda^{i}$ satisfying (19). We are to remember that $S_{i j} \lambda^{i} \lambda^{i}$ is positive when $S=$ const. is convex on its positive side and $S_{i j} \lambda^{i} \lambda^{j}$ is negative when $S=$ const. is concave on its positive side. It is easy to remember the sign if we bear (18) in mind.*

The interpretation of the last term in (15) is simple. If $\theta$ denotes the angle between the positive normals to $S=$ const. and $V=$ const., then

$$
S_{i} V^{i}=\cos \theta\left(S_{i} S^{i} \cdot V_{j} V^{j}\right)^{1 / 2}=\cos \theta \frac{\partial S}{\partial n_{S}} \frac{\partial V}{\partial n_{V}},
$$

when $\partial n_{S}, \partial n_{V}$ denote respectively elements of the arcs of the normals to $S=$ const., $V=$ const., drawn from the positive sides.

The research into the apsidal properties of a system resolves itself into a search for those regions of the $x$-space in which, for an assigned value of $E$, the apsidal function $\psi_{S}(x, \lambda, E)$ has definitely a positive or negative value for an arbitrary unit vector $\lambda^{i}$, or, more particularly, for a unit vector $\lambda^{i}$ tangent to $S=$ const. $\psi_{S}$ is, of course, bounded at any point, since

$$
a_{i j} \lambda^{i} \lambda^{j}=1 \text {. }
$$

The maximum and minimum values of $S_{i j} \lambda^{i} \lambda^{j}$ at a point for arbitrary directions are respectively the greatest and least of the roots of the determinantal equation

$$
\left|S_{i j}-\rho a_{i j}\right|=0
$$

which roots are all real. If we are only interested in directions tangential to $S$, we may introduce a special coördinate system in which $x^{N}=S$ and the parametric lines of $x^{N}$ are the orthogonal trajections of $S=$ const. Then, if Greek suffixes have a range from 1 to $N-1$, we have for any direction $\lambda^{i}$ tangential to $S=$ const.

$$
\psi_{S}(x, \lambda, E)=2(E-V) S_{\alpha \beta} \lambda^{\alpha} \lambda^{\beta}-a^{N N} \frac{\partial V}{\partial x^{N}}
$$

the maximum and minimum values of $S_{\alpha \beta} \lambda^{\alpha} \lambda^{\beta}$ are respectively the greatest and least roots of

$$
\left|S_{\alpha \beta}-\rho a_{\alpha \beta}\right|=0
$$

which roots are all real.

\footnotetext{
* The method adopted above differs from the method usually adopted in the discussion of curvature, being more convenient for the present purposes; cf. Eisenhart, Riemannian Geometry, 1926, p. 150 ,
} 
Every point of the manifold will belong to one of three regions or to the bounding surfaces between them. These regions are as follows:

(i) The region $R_{\min }$, in which $\psi_{\mathrm{S}}>0$ at each point for every direction tangent to $S=$ const. Every apse in $R_{\min }$ is a minimum apse.

(ii) The critical region in which at each point $\psi_{S}>0$ for some directions tangent to $S=$ const., and $\psi_{S}<0$ for other directions tangent to $S=$ const. The critical region sometimes reduces to a critical surface. When the system has only two degrees of freedom, and $\lambda^{i}$ is tangential to $S=$ const., $\lambda^{i}$ is then defined (except as to sign), and $\psi_{S}$ becomes merely a function of position: the critical region is then a curve. We shall see later (\$6) that when the manifold of configurations is flat, the critical region with respect to radial-apsides reduces to a surface.

(iii) The region $R_{\max }$, in which $\psi_{S}<0$ at each point for every direction tangent to $S=$ const. Every apse in $R_{\max }$ is a maximum apse.*

It is to be remembered that the apsidal properties to be discussed apply to all motions which possess a certain total energy $E$; when $E$ is altered, the apsidal properties will change, in general.

\section{PART II. RADIAL-APSIDES}

5. Radial-apsides in general. What precedes does not constitute an advance, as regards results, on the work of Hadamard; it consists chiefly of a translation into tensor notation of what is essential to the developments which follow.

We shall now consider radial-apsides, defined in $\$ 2$, in terms of the function $F$. This function, which I have called the "characteristic function" of the space, $\dagger$ is, at ordinary points of space, a regular function of the coördinates of $P, O$ being fixed, and is such that

$$
F_{i}=r \mu_{i}
$$

* When $S=V$, the regions $R_{\min }$ and $R_{\max }$ are related to, but not identical with, the attractive and repulsive regions of Hadamard (loc. cit., pp. 339, 360); our regions depend on the value of $E$; Hadamard's are independent of $E$.

$\dagger$ Proceedings of the London Mathematical Society, (2), vol. 32 (1931), pp. 241-258. At the time of writing that paper I did not know that this function had already been used by Hadamard in connection with the elementary solution of the general linear partial differential equation of the second order: cf. Hadamard, Lectures on Cauchy's Problem in Linear Partial Differential Equations, Yale, 1923, p. 89. It has also been employed by H. S. Ruse, Proceedings of the London Mathematical Society, (2), vol. 31 (1930), pp. 225-230, vol. 32 (1931), pp. 87-92, Proceedings of the Edinburgh Mathematical Society, (2), vol. 2 (1931), pp. 135-139. This function has long played an important part in the three-body problem: it is the function which appears in Jacobi's equation and in Sundman's inequality (cf. Birkhoff, Dynamical Systems, 1927, chapter ix; Whittaker, Analytical Dynamics, 1927, p. 342). 
where

$$
r=O P=(2 F)^{1 / 2},
$$

and $\mu^{i}$ is the unit tangent vector to $O P$ at $P$. We have also

$$
\begin{gathered}
(F)_{o}=0,\left(F_{i}\right)_{o}=0,\left(F_{i j}\right)_{o}=\left(a_{i j}\right)_{o},\left(F_{i j k}\right)_{o}=0, \\
\left(F_{i j k l}\right)_{O}=-\frac{1}{3}\left(R_{i k j l}+R_{i l j k}\right)_{o}
\end{gathered}
$$

where the covariant derivation is carried out with respect to the coördinates of $P, O$ being fixed, and the subscript $(O)$ indicates the limit as $P$ tends to $O$ : $R_{i j k l}$ is the curvature tensor with respect $a_{i j}{ }^{*}$ We note that, if $\lambda^{i}, \mu^{i}$ are two perpendicular unit vectors at $O$, then

$$
\left(F_{i j k l}\right)_{o} \lambda^{i} \lambda^{i} \mu^{k} \mu^{l}=-\frac{2}{3} K(\lambda, \mu),
$$

where $K(\lambda, \mu)$ is the Gaussian curvature corresponding to the two-space of the vectors.

For the discussion of radial-apsides we have (as particular cases of (16) and (15))

$$
\begin{aligned}
\frac{d^{2} F}{d t^{2}} & =\psi_{F}(x, \lambda, E), \\
\psi_{F}(x, \lambda, E) & =2(E-V) F_{i j} \lambda^{i} \lambda^{j}-F_{i} V^{i} \\
& =2(E-V) F_{i j} \lambda^{i} \lambda^{j}-r \frac{\partial V}{\partial r},
\end{aligned}
$$

where $\partial / \partial r$ refers to differentiation along the radial geodesic drawn from the point $O$. By (25), we may also write

$$
\psi_{F}(x, \lambda, E)=r\left\{2(E-V) k(F, \lambda)-\frac{\partial V}{\partial r}\right\},
$$

where $k(F, \lambda)$ is the curvature of the sphere $F=$ const. for the direction $\lambda^{i}$, counted positive when concave towards $O$. We shall suppose that $O$ is an ordinary point in the region for which $V<E$. Then it is evident that, for any assigned value of $E, \psi_{F}>0$ in the neighborhood of $O$. Since $\partial V / \partial r$ is the inward component of force along the radial geodesic, we may state the following result:

THEOREM I. For motion with total energy $E$, a region of minimum radialapsides with respect to a point $O$ in the region of motion $(V<E)$, not being a pole of $V$, extends out from $O$, including all those points at which the curvature of the

* The limits of the covariant derivatives of the 5th and 6th orders are also evaluated explicitly in the paper referred to. 
sphere with center $O$ (counted positive when concave towards $O$ ) is positive and greater than

$$
\frac{1}{2} \frac{R}{E-V}
$$

where $R$ is the component of force towards $O$ along the radial geodesic.

We shall now give a development in series which will be useful later. If $P$ ranges along a geodesic drawn from $O$, and has associated with it a direction $\lambda^{i}$ which is propagated parallelly along the geodesic as $P$ changes position, $\psi_{F}$ is a function of $r$, where $r=O P$. Expanding $\psi_{F}$ in a series, we find, using (33),

$$
\psi_{F}=a_{0}+a_{1} r+a_{2} r^{2}+\cdots
$$

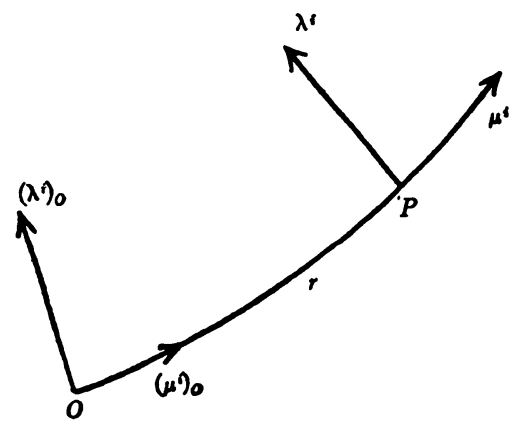

Fig. 1

where

$$
\begin{aligned}
& a_{0}= 2(E-V)_{o}, \quad a_{1}=-3\left(\frac{\partial V}{\partial r}\right)_{o} \\
& a_{2}=-2\left(\frac{\partial^{2} V}{\partial r^{2}}\right)_{o}+(E-V)_{o}\left(F_{\left.i j k_{1} k_{2} \lambda^{i} \lambda^{j} \mu^{k_{1}} \mu^{k_{2}}\right)_{o},}\right. \\
& a_{n}=-\frac{n+2}{n !}\left(\frac{\partial^{n} V}{\partial r^{n}}\right)_{o}-\frac{2}{n !}\left\{\frac{n(n-1)}{2 !} \frac{\partial^{n-2} V}{\partial r^{n-2}} F_{i j k_{1} k_{2}} \lambda^{i} \lambda^{j} \mu^{k_{1}} \mu^{k_{2}}+\cdots\right. \\
&\left.+n \frac{\partial V}{\partial r} F_{i j k_{1} \cdots k_{n-1}} \lambda^{i} \lambda^{j} \mu^{k_{1}} \cdots \mu^{k_{n-1}}-(E-V) F_{i j k_{1} \cdots k_{n}} \lambda^{i} \lambda^{i} \mu^{k_{1}} \cdots \mu^{k_{n}}\right\}_{O} \\
& \quad(n=3,4, \cdots),
\end{aligned}
$$

where, as above, $\mu^{i}$ is the unit tangent vector to the geodesic $O P$, and the subscript $(O)$ indicates evaluation at $O$. The coefficients $a_{0}, a_{1}$ are functions only of the direction of $\lambda^{i}$ at $O ; a_{2}, a_{3}, \cdots$ also involve the direction $\left(\lambda^{i}\right)_{o}$, which will be taken perpendicular to $O P$ but otherwise arbitrary. If $\left(\mu^{i}\right)_{0}$ are fixed, and $\left(\lambda^{i}\right)_{o}$ given arbitrary values, subject to $\left(\lambda_{i} \lambda^{i}\right)_{o}=0$ and $\left(\mu_{i} \lambda^{i}\right)_{o}=0$, each of 
these coefficients has a lower and an upper bound, which we may denote by a single and a double accent respectively. Thus we have

$$
a_{0}+a_{1} r+a_{2}^{\prime} r^{2}+\cdots \leqq \psi_{F} \leqq a_{0}+a_{1} r+a_{2}^{\prime \prime} r^{2}+\cdots .
$$

As we pass out from $O$ along a radial geodesic, we start in a region $R_{\min }$, which extends up to and perhaps beyond the surface whose polar equation is

$$
a_{0}+a_{1} r+a_{2}^{\prime} r^{2}+\cdots=0
$$

we then pass into the critical region, from which we emerge into the region $R_{\max }$ before or when we cross the surface

$$
a_{0}+a_{1} r+a_{2}^{\prime \prime} r^{2}+\cdots=0 .
$$

The surfaces (40), (41) include, but are not necessarily the bounding surfaces of, the critical region in which the apsides may be of either type. We observe that when the space is flat (so that the $F$ 's in (38) all vanish), the surfaces (40) and (41) coincide and the critical region reduces to a critical surface. We shall discuss this in the next section.

6. Radial-apsides for flat manifolds and for radial manifolds. Let us note a remarkable property of flat manifolds, for which it is known that

$$
F_{i j}=a_{i j}
$$

We have then

$$
\psi_{F}(x, \lambda, E)=2(E-V)-F_{i} V^{i}
$$

or

$$
\psi_{F}(x, \lambda, E)=2(E-V)-r \frac{\partial V}{\partial r}
$$

$\partial V / \partial r$ being taken along the geodesic $O P$. We observe that, in this case, $\psi_{F}$ does not depend on $\lambda^{i}$ : it is mere function of position. Flat manifolds of configurations are of considerable dynamical importance; they include the manifold corresponding to a system consisting of any number of free particles. We may state the following theorem:

THEOREM II. If the manifold of configuration is flat, and $r$ represents the geodesic distance from a fixed point $O$, all the points at which $r$ can take a minimum value in the course of a motion with total energy $E$ are situated in the region $R_{\min }$, for which

$$
\psi_{F} \equiv 2(E-V)-r \frac{\partial V}{\partial r}>0
$$


and all the points at which $r$ can take a maximum value are situated in the region $R_{\max }$ for which

$$
\psi_{F} \equiv 2(E-V)-r \frac{\partial V}{\partial r}<0
$$

the two regions being separated by a critical surface or surfaces

$$
\psi_{F} \equiv 2(E-V)-r \frac{\partial V}{\partial r}=0 .
$$

I have defined a radial manifold ${ }^{*}$ as one possessing geometrical isotropy with respect to a point $O$. It is characterised by a single function $f(r)$, which is the ratio of the normal distance $\eta$ between neighboring radial geodesics at a distance $r$ from $O$ to the infinitesimal angle $\delta \phi$ between their initial directions at $O$, so that

$$
\eta=f(r) \delta \phi .
$$

(A manifold of this type is associated with the motion of a particle on a smooth surface of revolution.) For such a manifold, we have

$$
r_{i j}=\frac{f^{\prime}(r)}{f(r)}\left(a_{i j}-r_{i} r_{j}\right)
$$

and therefore, since

$$
2 F=r^{2}, F_{i}=r r_{i}, F_{i j}=r r_{i j}+r_{i} r_{j},
$$

we have

$$
F_{i j} \lambda^{i} \lambda^{j}=\frac{r f^{\prime}}{f}\left\{1-\left(r_{i} \lambda^{i}\right)^{2}\right\}+\left(r_{i} \lambda^{i}\right)^{2}=\frac{r f^{\prime}}{f}+\left(1-\frac{r f^{\prime}}{f}\right)\left(r_{i} \lambda^{i}\right)^{2} .
$$

At a point $P$ the maximum value of $\left(r_{i} \lambda^{i}\right)^{2}$ is 1 , corresponding to the direction of $O P$ at $P$, and also to the reversed direction; the minimum value is zero, corresponding to a direction perpendicular to $O P$. Thus for directions at $P$ tangential to $F=$ const., we have

$$
\begin{aligned}
\psi_{F}(x, \lambda, E) & =2(E-V) r f^{\prime} / f-F_{i} V^{i} \\
& =r\left\{2(E-V) f^{\prime} / f-\partial V / \partial r\right\} .
\end{aligned}
$$

* Hodographs of general dynamical systems, Transactions of the Royal Society of Canada, (3), vol. 25, Section III (1931), p. 132; the radial manifold includes the manifold of constant curvature as a special case. 
Accordingly we may state the following result:

THEOREM III. If the manifold of configuration is radial with respect to $O$, and $r$ represents the geodesic distance from $O$, all the points at which $r$ can take a minimum value are situated in the region $R_{\min }$ for which

$$
\psi_{F} \equiv 2(E-V) f^{\prime} / f-\partial V / \partial r>0,
$$

and all the points for which $r$ can take a maximum value are situated in the region $R_{\max }$ for which

$$
\psi_{F} \equiv 2(E-V) f^{\prime} / f-\partial V / \partial r<0,
$$

the two regions being separated by a critical surface or surfaces

$$
2(E-V) f^{\prime} / f-\partial V / \partial r=0 .
$$

It should be remarked that, while $\partial V / \partial r$ is a function of position, it is not, of course, a function of $r$ only in general.

For a flat space, $f=r$, and Theorem III reduces to Theorem II.

7. Radial-apsides for flat manifolds with homogeneous potential energy. Supposing the manifold of configurations to be flat, let us take normal coordinates such that

$$
T=\frac{1}{2}\left\{\left(\dot{x}^{1}\right)^{2}+\left(\dot{x}^{2}\right)^{2}+\cdots+\left(\dot{x}^{N}\right)^{2}\right\} .
$$

Let the point $O$ be the origin of the coördinates. We have then

$$
F=\frac{1}{2} r^{2}=\frac{1}{2}\left\{\left(x^{1}\right)^{2}+\left(x^{2}\right)^{2}+\cdots+\left(x^{N}\right)^{2}\right\} .
$$

Let us suppose that $V$ is a homogeneous function of degree $n$ in these coördinates. (The arbitrary constant always associated additively with $V$ is therefore chosen to make $V$ vanish at infinity if $n<0$ and to make $V$ vanish at $O$ if $n>0$.) We have then

$$
x^{i} \frac{\partial V}{\partial x^{i}}=n V,
$$

and, since $F^{i}=F_{i}=x^{i}$,

$$
F_{i} V^{i}=r \frac{\partial V}{\partial r}=n V .
$$

As we proceed along a radial geodesic, we have

$$
V=A r^{n}
$$

where $A$ is a constant along the geodesic, but changes.in general when we pass to a different radial geodesic. The systems under consideration might have been defined by the invariant relation (59), or by (60). 
As we pass out along a radial geodesic from $O$, the ratios of the coördinates remain fixed, and consequently, since $V_{i}$ are homogeneous of degree $(n-1)$ in the coördinates, we may put

$$
V_{i}=B_{i} r^{n-1},
$$

where $B_{i}$ is a constant vector for an assigned radial geodesic; by (59) and (60),

$$
B_{i} F^{i}=n A r .
$$

Thus, along an assigned radial geodesic, the direction of the intensity $\left(-V^{i}\right)$ remains parallel to a fixed direction, and the magnitude of the intensity $\left(V_{i} V^{i}\right)^{1 / 2}$ varies as $r^{n-1}$. By (59), the intensity at $P$ makes an acute angle with $O P$ if $n V$ is negative, an obtuse angle if $n V$ is positive, and a right angle if $n V$ is zero.

For a system of the type considered we have, by (44) and (59),

$$
\psi_{F}(x, \lambda, E)=2(E-V)-n V=2 E-(n+2) V .
$$

The critical surface, which separates minimum-apsides from maximumapsides, is therefore, if it exists, the equipotential surface

$$
V=\frac{2 E}{n+2} .
$$

The fact that the kinetic energy cannot be negative restricts the motion to the region for which

$$
E-V \geqq 0 .
$$

This inequality may, of course, be satisfied throughout the whole manifold, in which case there is no restriction. In order that the critical surface (64) may lie in the region in which (65) is satisfied, it is necessary and sufficient that

$$
\frac{n E}{n+2} \geqq 0 \text {. }
$$

Let us suppose that $E$ is assigned, and that, travelling a distance $r_{E}$ along a geodesic from $O$, we encounter the surface $V=E$. If the potential energy is $V$ at a point on the same geodesic at a distance $r$ from $O$, we have

$$
\frac{V}{E}=\frac{r^{n}}{r_{E^{n}}} \text {. }
$$

Hence the critical surface $\psi=0$ has the polar equation

$$
r=r_{E}\left(\frac{2}{n+2}\right)^{1 / n} .
$$


The critical surface is therefore generated from the surface $V=E$ by a uniform expansion with respect to $O$ in the ratio

$$
\rho_{n}=\left(\frac{2}{n+2}\right)^{1 / n},
$$

of which we note the values in the two important cases $n=-1$ and $n=2$ as respectively

$$
\rho_{-1}=\frac{1}{2}, \quad \rho_{2}=\frac{1}{2^{1 / 2}} .
$$

Since, in the classical theory of small oscillations, the manifold is flat and the potential energy homogeneous of the second degree, to the order considered, we may state the following remarkable result:

THEOREM IV. When a system performs small oscillations about a position of stable equilibrium, with total energy $E$, there exists inside the equipotential ellipsoid $V=E$, beyond which the system cannot pass, another equipotential ellipsoid, similar and similarly situated, with axes reduced in the ratio $1: 2^{1 / 2}$, such that all the minimum radial-apsides with respect to the position of equilibrium are situated inside this ellipsoid, and all the maximum radial apsides in the homoeoid bounded by this ellipsoid and $V=E$.

Before proceeding to a classification, according to the value of the degree of homogeneity $n$, we may state our general results in the following form:

THEOREM V. When a system with a flat manifold of configurations and a potential energy $V$, which is homogeneous of degree $n$ in the normal coördinates, moves with a total energy $E$, the critical surface which divides the regions of minimum and maximum radial-apsides has the equation

$$
V=\frac{2 E}{n+2}
$$

for all directions in which geodesics from $O$ meet the surface $V=E$, the critical surface is generated by measuring off from $O$ along such geodesics, a distance equal to the distance from $O$ to $V=E$, multiplied by the constant $\rho_{n}$, given by (69); the polar equation for the critical surface has the form (68).

It seems best to classify the results according to the value of $n$. We shall accordingly consider the five cases:

(a) $n<-2$. Example: a particle under a central force, varying as the inverse fourth power of the distance $(n=-3)$.

(b) $n=-2$. Examples: a particle under a central force, varying as the inverse cube of the distance; an electrified particle moving under the attraction of a fixed dipole. 
(c) $-2<n<0$. Examples: a particle under a central force, varying as the inverse square of the distance; the two-body problem; the three-body problem $(n=-1$ in each case).

(d) $n=0$. Trivial examples: a free particle; a lamina moving on a smooth plane under no forces. Examples: a particle in a plane acted on by a force perpendicular to the radius vector, of magnitude $(k \cos \theta) / r$, where $\theta$ is the polar angle; a particle in a plane under a transverse force $k / r$ (since $V$ is multiple-valued, a Riemann surface must be taken for the manifold of configurations).

(e) $0<n$. Examples: a particle under gravity on a smooth circular cone with axis vertical $(n=1)$; a particle in a plane under a central force varying as the distance $(n=2)$; small oscillations about equilibrium (approximate theory) $(n=2)$; small oscillations about the lowest point of a smooth surface whose equation is $z=x^{4}+y^{4}$ (approximate theory) $(n=4)$.

A systematic treatment is assisted by a diagram (Fig. 2). Let us take rectangular coördinates to represent $E$ and $V$. To any given state of the

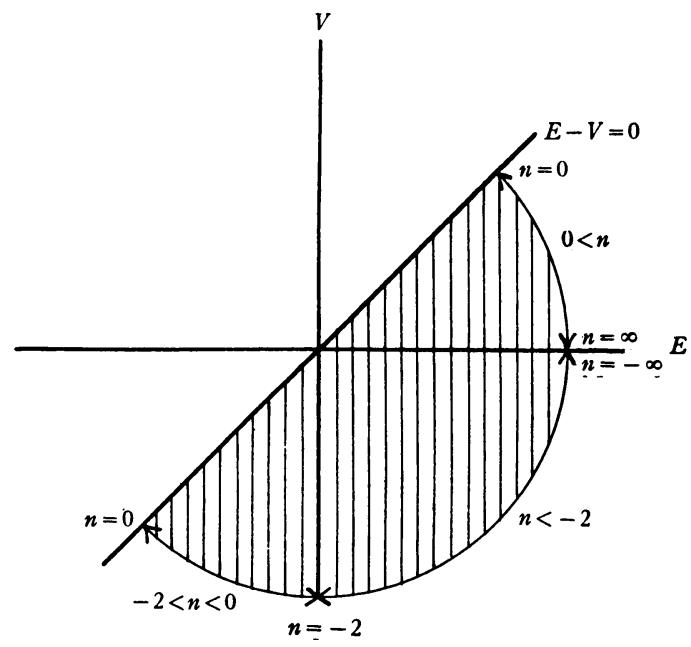

Fig. 2

system there will correspond a point on the $(E, V)$ plane: but, of course, to a point on the plane there will correspond a multiplicity of states. If we are told the state of a system at an instant, the points on the $(E, V)$ plane corresponding to its subsequent history will lie on a line $E=$ const. These lines (drawn in the figure) are bounded above by the line $E-V=0$. The portion of the plane above this line is forbidden to the system, and is therefore left blank. Other portions may also be forbidden. For example, the potential en- 
ergy may be negative throughout, as in the three-body problem. We would then have to leave blank the whole of the plane above $V=0$.

As to the position of the line $\psi_{F}=0$ or (71), we observe that it passes into the region $E-V>0$ as follows:

into the fourth quadrant if $n<-2$;

into the third quadrant if $-2<n<0$;

into the first quadrant if $0<n$.

These regions are indicated in the figure.

It follows from (63) that the region lying to the right of the ray $\psi_{F}=0$ (drawn into the shaded region) is $R_{\min }$ (the region of minimum $F$-apsides); that to the left is $R_{\max }$ (the region of maximum $F$-apsides).

The figures which follow illustrate the five cases considered. When the motion may be oscillatory, the corresponding line $E=$ const. is marked - - - ;

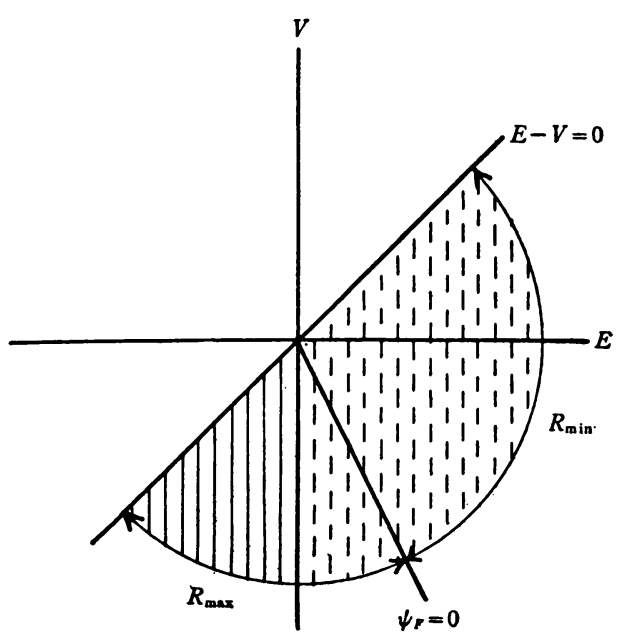

Fig. 3

$n<-2$.

$(n=-3$ shown.)

when the motion can have at most one apse, the line is drawn full; whether it is a maximum or minimum apse will be determined by the infinite sector $\left(R_{\max }\right.$ or $\left.R_{\min }\right)$ in which it is contained. A motion with at most one apse may be called "non-oscillatory."

If, when a portion of the plane has to be excluded on account of special knowledge (e.g. $V<0$ ), the excluded portion contains the line $\psi_{F}=0$, then the lines marked - - - in the part of the figure not left blank must be changed to full lines to indicate non-oscillatory motion. 


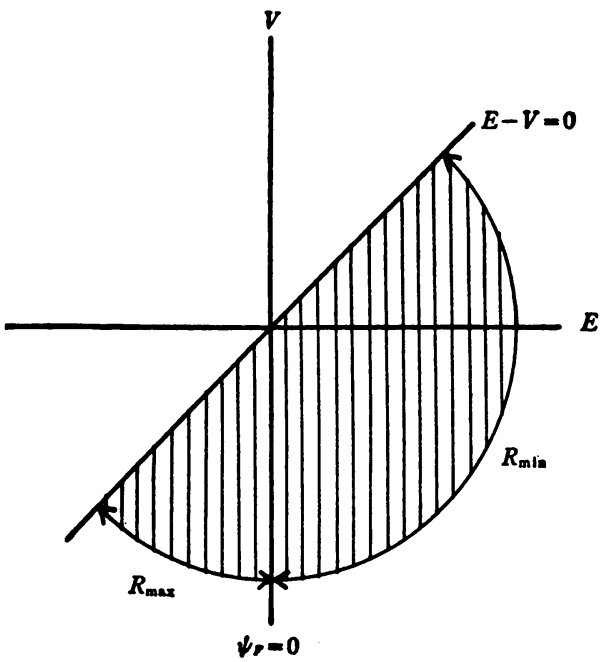

Fig. 4

$n=-2$.

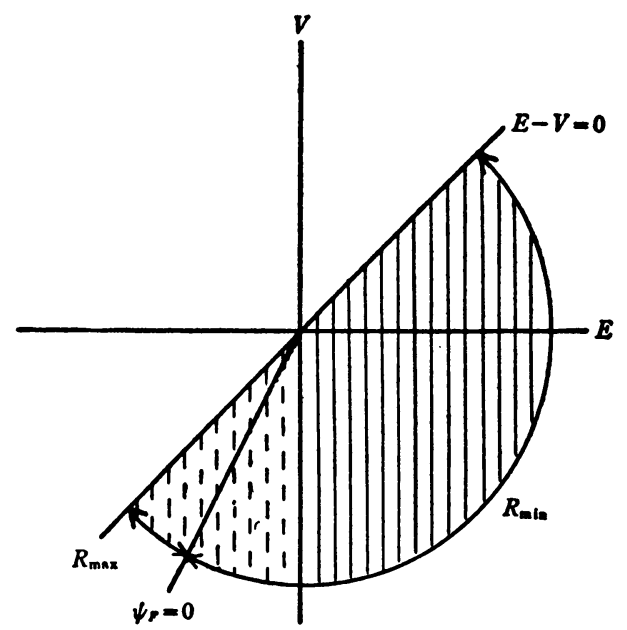

Fig. 5

$-2<n<0$.

( $n=-1$ shown.)

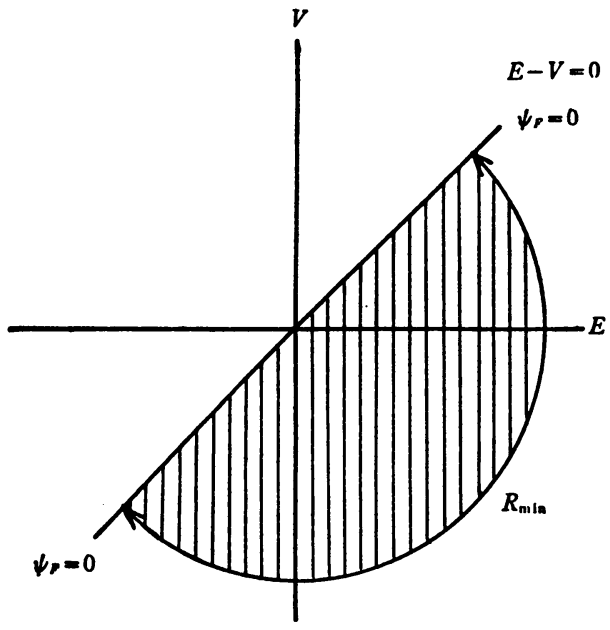

Fig. 6 $n=0$.

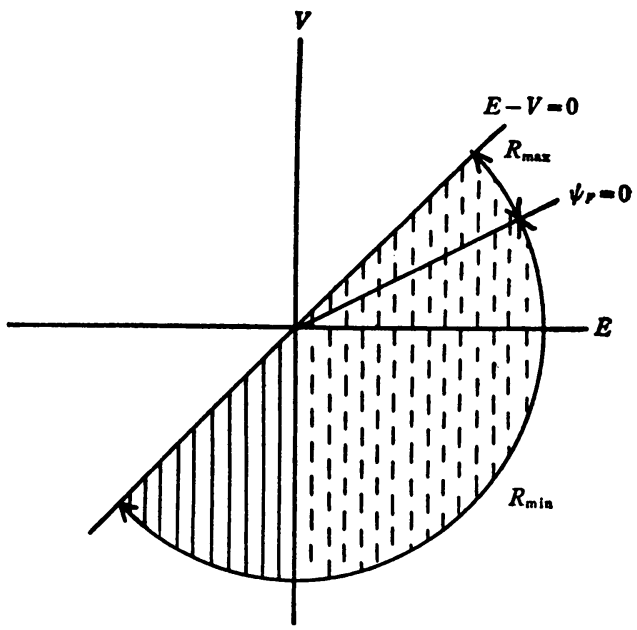

Fig. 7 $0<n$. ( $n=2$ shown.) 
An interesting feature of the foregoing diagrams is that it is possible to proceed so far with the classification without differentiating between attractive and repulsive fields. But a field that can be called "attractive" or "repulsive" is of quite a special character. In the general case, the field of force will be attractive for some radii drawn from $O$ in the configuration space and repulsive for others. Thus if $n>0$, we shall have $V=0$ at $O$, and at infinity one of the three values $V=-\infty, V=0, V=+\infty$ according to direction. In the case of any number of bodies, attracting or repelling one another according to the inverse square law, we have $n=-1$, and $V=0$ at infinity in both cases: for attraction $V<0$, and for repulsion $V>0$. Thus the diagrams for these two cases are as follows:

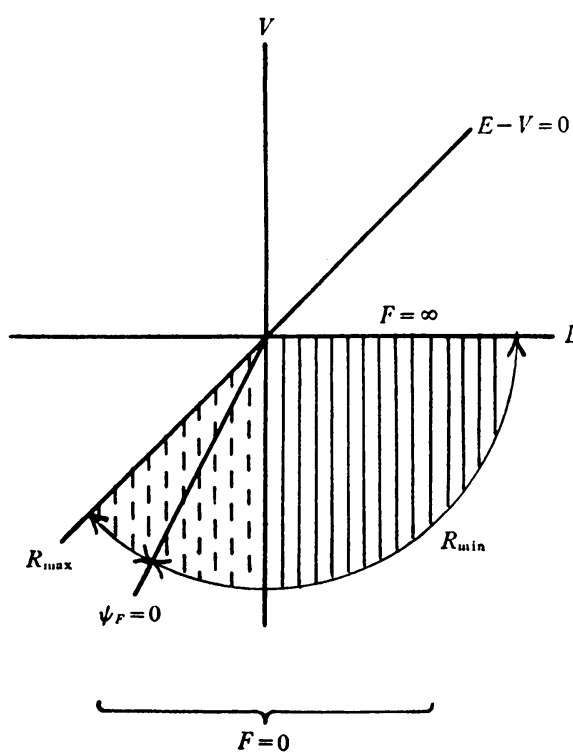

Fig. 8

Any number of bodies attracting one another according to the inverse square law $(n=-1)$.

An important class of motions consists of those for which $F=$ const. It includes the circular orbits under central forces and the steady motions in the three-body problem, the point $O$ corresponding to coincidence of the bodies at their common center of mass, supposed stationary. For such motions we must have $\psi_{F}=0$ permanently, and since, except in the case $n=-2$, the line $\psi_{F}=0$ cuts $E=$ const. at one point only, $V$ must be constant for such a motion. Hence we may state the following result:

THEOREM VI. For a system with a flat manifold of configurations and a potential energy homogeneous of degree $n(n \neq-2)$ in the normal coordinates, any 
motion for which the distance from $O$ is constant (every point a radial-apse) must take place on the equipotential surface given by $\psi_{F}=0$ or

$$
V=\frac{2 E}{n+2} .
$$

If $n=-2$, such motions must possess zero total energy $(E=0)$.

It is perhaps not out of place here to point out a geometrical reason why the problem of three bodies belongs to a class different from that of the problem of two bodies. In each case, making $O$ correspond to complete collision at the center of mass, supposed at rest, the motions take place in flat submanifolds of $9-3=6$ and $6-3=3$ dimensions, respectively. From the fixation of the center of mass it is possible to express $V$ for the two bodies as a function of $F$. Hence the equipotential surfaces $V=$ const. are spheres $F=$ const., and we are dealing with the generalised case of a particle under a central force depending only on the distance. In the case of the problem of three bodies, the surfaces $V=$ const. and $F=$ const. no longer coincide: we have to deal with the generalisation of the motion of a particle in a plane under a force which is not central, but has a transverse component.

8. Radial-apsides for motion near a position of instantaneous rest. Let us now return to the general case, in which the manifold of configurations is no longer flat, and in which there is no restriction on the form of the potential energy.

A position of instantaneous rest must lie on the surface $V=E$. Let us choose such a position as the point $O$, from which $F$ is measured. The $F$ apsidal function (35) is

$$
\psi_{F}=2(E-V) F_{i j} \lambda^{i} \lambda^{i}-F_{i} V^{i}=2(E-V) F_{i j} \lambda^{i} \lambda^{i}-r \frac{\partial V}{\partial r},
$$

where, it will be remembered, $\lambda^{i}$ is the unit tangent vector to the orbit, $r=O P$, and $\partial / \partial r$ denotes differentiation along $O P$.

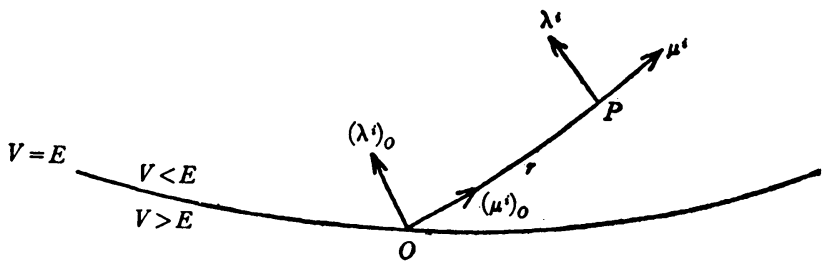

Fig. 10

Let us investigate the value of $\psi_{F}$ at $P$. Let $\mu^{i}$ be the unit tangent vector 
at $P$ to the geodesic $O P$. By propagating $\lambda^{i}$ parallelly along $O P$, we can assign to each point of $O P$ a definite value of $\psi_{F}$ corresponding to an assigned direction $\lambda^{i}$ at $P$. We can then develop $\psi_{F}$ as a power series in $r$, provided that $P$ is sufficiently close to $O$ to ensure the convergence of the series. Thus we find

$$
\psi_{F}=a_{1} r+a_{2} r^{2}+a_{3} r^{3}+\cdots,
$$

where the coefficients are found by putting $(E-V)_{o}=0$ in (38), being therefore

$$
\begin{aligned}
& a_{1}=-3\left(V_{i} \mu^{i}\right)_{o}=-3\left(\frac{\partial V}{\partial r}\right)_{o} \\
& a_{2}=-2\left(V_{i j} \mu^{i} \mu^{j}\right)_{O}=-2\left(\frac{\partial^{2} V}{\partial r^{2}}\right)_{0}, \\
& a_{3}=-\frac{5}{6}\left(\frac{\partial^{3} V}{\partial r^{3}}\right)_{o}-\left(\frac{\partial V}{\partial r} F_{i j k_{1} k_{2} \lambda^{i} \lambda^{j} \mu^{k_{1}} \mu^{k_{2}}}\right)_{o} \\
& a_{n}=-\frac{n+2}{n !}\left(\frac{\partial^{n} V}{\partial r^{n}}\right)_{o} \\
& -\frac{2}{n !}\left\{\frac{n(n-1)}{2 !} \frac{\partial^{n-2} V}{\partial r^{n-2}} F_{i j k_{1} k_{2}} \lambda^{i} \lambda^{i} \mu^{k_{1}} \mu^{k_{2}}+\cdots\right. \\
& \left.+n \frac{\partial V}{\partial r} F_{i j k_{1} \cdots k_{n-1}} \lambda^{i} \lambda^{i} \mu^{k_{1}} \cdots \mu^{k_{n-1}}\right\}_{o} \quad(n=4,5, \cdots),
\end{aligned}
$$

where the subscript $(O)$ indicates evaluation at $O$. We observe that the first two terms are independent of the curvature of the manifold, as represented by $F_{i j k_{1} k_{2}}$, etc. As we go out along the normal to the surface $V=E$ into the region of motion $(V<E)$, we have

$$
\left(\frac{\partial V}{\partial r}\right)_{o}<0 .
$$

Therefore $a_{1}$ is positive for this direction, and therefore $\psi_{F}>0$ on the geodesic normal to $V=E$, in the vicinity of $O$. This is true, not only for the normal, but for every other geodesic drawn from $O$ into the region $V<E$, provided that it lies on the same side of the tangent geodesic surface at $O$. Thus the critical region, if it extends up to $O$, must reduce at $O$ to a surface touching $V=E$ there. 


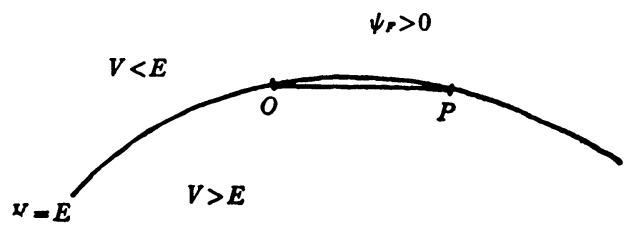

Fig. 11

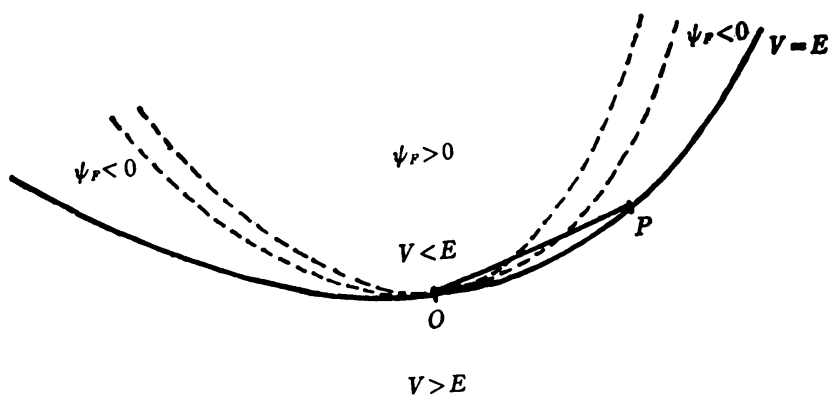

Fig. 12

Let us examine the sign of $\psi_{F}$ on $V=E$ near $O$. If $P$ is on $V=E$, then

$$
0=\left(\frac{\partial V}{\partial r}\right)_{o} r+\frac{1}{2}\left(\frac{\partial^{2} V}{\partial r^{2}}\right)_{o} r^{2}+\cdots ;
$$

adding this equation, multiplied by 3 , to (73), we get

$$
\psi_{F}=-\frac{1}{2}\left(\frac{\partial^{2} V}{\partial r^{2}}\right)_{o} r^{2}+\cdots,
$$

showing that $\psi_{F}$ has the opposite sign to $\left(\partial^{2} V / \partial r^{2}\right)_{o}$. Three cases may arise as to the nature of the surface $V=E$ at $O$ :

(i) The surface $V=E$ is convex towards the region of motion $(V<E)$, for every tangential direction at $O$ (Fig. 11); then $\left(\partial^{2} V / \partial r^{2}\right)_{O}<0$ for every tangential direction at $O$, and hence $\psi_{F}>0$ on the surface $V=E$ near $O$ and in the part of the region $V<E$ near $O$. The critical region near $O$ in fact lies on the side $V>E$, and does not affect the motion. Hence the region $V<E$ near $O$ is a region of minimum $F$-apsides, and this region extends to the surface having the polar equation

$$
a_{1}+a_{2} r+a_{3}^{\prime} r^{2}+\cdots=0,
$$

where $a_{3}^{\prime}, a_{4}^{\prime}, \cdots$ are the lower bounds of $a_{3}, a_{4}, \cdots$ for arbitrary directions $\lambda^{i}$ at $O$, perpendicular to the direction in which the geodesic along which $r$ is measured is drawn; the more distant boundary of the critical region lies not 
further from $O$ than the surface having the polar equation

$$
a_{1}+a_{2} r+a_{3}^{\prime \prime} r^{2}+\cdots=0,
$$

where $a_{3}^{\prime \prime}, a_{4}^{\prime \prime}, \cdots$ are upper bounds.

(ii) The surface $V=E$ is concave towards the region of motion $(V<E)$, for every tangential direction at $O$ (Fig. 12); then $\left(\partial^{2} V / \partial r^{2}\right)_{O}>0$ for every tangential direction at $O$, and hence $\psi_{F}<0$ on the part of the surface $V=E$ near $O$. As we pass through the region $V<E$ from a point on $V=E$ to a point on the normal, $\psi_{F}$ changes sign from - to + . Accordingly there is a critical region in $V<E$, whose bounding surfaces (represented by the dotted lines in Fig. 12) both touch $V=E$ at $O$, and which is contained between the surfaces (78), (79). The region of minimum $F$-apsides is that which contains the normal to $V=E$ at $O$, and the region of maximum $F$-apsides is the narrowing region adjacent to $V=E$.

(iii) The surface $V=E$ is convex towards the region of motion $(V<E)$ for some tangential directions and concave for others. A critical region will exist in $V<E$ in the neighborhood of $O$, but only approaching $O$ in those sections which have their concavity towards $V<E$.

The above circumstances are easily illustrated by a particle on a smooth sphere under gravity, or a particle in a plane attracted to a center by a force varying as the distance. These being two-dimensional systems, the critical region is a curve, which in the latter case is a circle touching the circle $V=E$ internally at $O$, and having a diameter equal to $3 / 4$ of the diameter of the circle $V=E$.

9. Radial-apsides for motion near a position of equilibrium. Taking $O$ at a position of equilibrium, we have

$$
\left(V_{i}\right)_{o}=0,
$$

and therefore

$$
\left(\frac{\partial V}{\partial r}\right)_{o}=0,
$$

for every geodesic drawn from $O$. By (35),

$$
\psi_{F}=2 E F_{i j} \lambda i \lambda r-2 V F_{i j} \lambda^{i} \lambda^{j}-r \frac{\partial V}{\partial r} .
$$

Let us assume, without loss of generality, that $V$ vanishes at $O$, so that

$$
(V)_{o}=0 \text {. }
$$

Then, expanding $\psi_{F}$, we obtain 


$$
\psi_{F}=E\left(a_{0}+a_{2} r^{2}+a_{3} r^{3}+\cdots\right)+b_{2} r^{2}+b_{3} r^{3}+\cdots,
$$

where

$$
\begin{aligned}
& a_{0}=2 \text {, } \\
& a_{2}=\left(F_{i j k_{1} k_{2}} \lambda^{i} \lambda^{i} \mu^{k_{1}} \mu^{k_{2}}\right)_{O}, \\
& a_{n}=\frac{2}{n !}\left(F_{i j k_{1}} \cdots k_{n} \lambda^{i} \lambda^{\left.j \mu^{k_{1}} \cdots \mu^{k_{n}}\right)_{o}} \quad(n=3,4, \cdots)\right. \text {; } \\
& b_{2}=-2\left(\frac{\partial^{2} V}{\partial r^{2}}\right) \\
& b_{3}=-\frac{5}{6}\left(\frac{\partial^{3} V}{\partial r^{3}}\right)_{0} \\
& b_{4}=-\frac{1}{4}\left(\frac{\partial^{4} V}{\partial r^{4}}\right)_{o}-\frac{1}{2}\left(\frac{\partial^{2} V}{\partial r^{2}} F_{i j k_{1} k_{2}} \lambda^{i} \lambda^{j} \mu^{k_{1}} \mu^{k_{2}}\right)_{O}, \\
& b_{n}=-\frac{2}{n !}\left\{\frac{n+2}{2} \frac{\partial^{n} V}{\partial r^{n}}+\frac{n(n-1)}{2 !} \frac{\partial^{n-2} V}{\partial r^{n-2}} F_{i j k_{1} k_{2}} \lambda^{i} \lambda^{i} \mu^{k_{1}} \mu^{k_{2}}+\cdots\right. \\
& \left.+\frac{n(n-1)}{2 !} \frac{\partial^{2} V}{\partial r^{2}} F_{i j k_{1} \cdots k_{n-2}} \lambda^{i} \lambda^{j} \mu^{k_{1}} \cdots \mu^{k_{n-2}}\right\}_{0} \quad(n=5,6, \cdots) \text {; }
\end{aligned}
$$

where, as before, the subscript $(O)$ indicates evaluation at $O, \lambda^{i}$ is propagated parallelly along the geodesic $O P$, and $\mu^{i}$ is the unit tangent vector to the geodesic.

Having assigned the unit vector $\left(\lambda^{i}\right)_{O}$, then, as we pass out along a geodesic from $O, \psi_{F}$ is a function of $r$ only. $\psi_{F}$ starts with the value $2 E$. If it vanishes for some value of $r$, we shall have, for that value of $r$,

$$
E=-r^{2} \frac{b_{2}+b_{3} r+\cdots}{a_{0}+a_{2} r^{2}+\cdots} .
$$

$E$ can be expanded in a power series in $r$, which will start with the power $r^{2}$ if $b_{2} \neq 0$, and with the power $r^{3}$ if $b_{2}=0, b_{3} \neq 0$. Let us assume that

$$
b_{2}=-2\left(\frac{\partial^{2} V}{\partial r^{2}}\right)_{0} \neq 0,
$$

for the geodesic under consideration. We have then from (86)

$$
E=r^{2}\left(c_{0}+c_{1} r+c_{2} r^{2}+\cdots\right) \text {, }
$$

where

$$
c_{0}=-b_{2} / a_{0}=\left(\partial^{2} V / \partial r^{2}\right)_{o}
$$


Let us first consider the case where $O$ is a position of stable equilibrium, and

$$
\left(\frac{\partial^{2} V}{\partial r^{2}}\right)_{O}>0
$$

for all geodesics from $O$. We shall consider only motions which pass into the surrounding region, $\dot{V}>0$; for these motions $E>0$. Equation (88) then gives

$$
\left(\frac{E}{c_{0}}\right)^{1 / 2}= \pm r\left(1+\frac{1}{2} \frac{c_{1}}{c_{0}} r+\cdots\right) .
$$

This series has only one reversion which gives a positive $r$ tending to zero as $E$ tends to zero. It will be of the form

$$
r=\left(\frac{E}{c_{0}}\right)^{1 / 2}\left[1+d_{1}\left(\frac{E}{c_{0}}\right)^{1 / 2}+d_{2} \frac{E}{c_{0}}+\cdots\right] .
$$

Here the coefficients $d_{1}, d_{2}, \cdots$ depend only on the initial direction of the geodesic at $O$ and the initial values $\left(\lambda^{i}\right)_{o}$. Their values are most easily found by substitution in the equation

$$
E\left(a_{0}+a_{2} r^{2}+\cdots\right)+b_{2} r^{2}+b_{3} r^{3}+\cdots=0 .
$$

We find, for the first two coefficients, remembering (34),

$$
\begin{aligned}
& d_{1}=-\frac{5}{24}\left(\frac{\partial^{3} V / \partial r^{3}}{\partial^{2} V / \partial r^{2}}\right)_{o} \\
& d_{2}=-\frac{1}{12}(K(\lambda, \mu))_{o}+\frac{125}{1152}\left(\frac{\partial^{3} V / \partial r^{3}}{\partial^{2} V / \partial r^{2}}\right)_{o}^{2}-\frac{1}{16}\left(\frac{\partial^{4} V / \partial r^{4}}{\partial^{2} V / \partial r^{2}}\right) .
\end{aligned}
$$

The region $V<E$ may contain critical regions other than that which is yielded by consideration of (92); but they will not tend to $O$ as $E$ tends to zero, and therefore may be excluded from the region $V<E$ by making $E$ sufficiently small. To obtain these other regions, we note that, as $E$ tends to zero, then for an assigned geodesic from $O$ and assigned $\left(\lambda^{i}\right)_{o}, \psi_{F}$ tends to zero for those values of $r$ which satisfy

$$
b_{2}+b_{3} r+b_{4} r^{2}+\cdots=0 .
$$

If $r=r_{1}$ is a solution of this equation, the value of $r$ for which $\psi_{F}=0$ will have an expression of the form

$$
r=r_{1}+e_{1} E+e_{2} E^{2}+\cdots,
$$

where $r_{1}, e_{1}, e_{2}, \cdots$ depend only on the direction of the geodesic at $O$ and the 
unit vector $\left(\lambda^{i}\right)_{o}$ perpendicular to that direction. The corresponding critical region lies between the surfaces

$$
\begin{aligned}
& r=r_{1}^{\prime}+e_{1}^{\prime} E+e_{2}^{\prime} E^{2}+\cdots, \\
& r=r_{1}^{\prime \prime}+e_{1}^{\prime \prime} E+e_{2}^{\prime \prime} E^{2}+\cdots,
\end{aligned}
$$

where $r_{1}^{\prime}, e_{1}^{\prime}, e_{2}^{\prime}, \cdots, r_{1}^{\prime \prime}, e_{1}^{\prime \prime}, e_{2}^{\prime \prime}, \cdots$ denote respectively minimum and maximum values for arbitrary choice of the unit vector $\left(\lambda^{i}\right)_{o}$ perpendicular to the geodesic at $O$. We may state the result

THEOREM VII. When a system performs finite oscillations about a position of stable equilibrium $O$ (for which $V=0$ and (90) is true), the total energy $E$ being sufficiently small but finite, the region $V<E$ contains a single critical region, lying between the surface whose polar equation is

$$
r=\left(\frac{E}{c_{0}}\right)^{1 / 2}\left[1+d_{1}\left(\frac{E}{c_{0}}\right)^{1 / 2}+d_{2}^{\prime} \frac{E}{c_{0}}+d_{3}^{\prime}\left(\frac{E}{c_{0}}\right)^{3 / 2}+\cdots\right]
$$

and the surface whose polar equation is

$$
r=\left(\frac{E}{c_{0}}\right)^{1 / 2}\left[1+d_{1}\left(\frac{E}{c_{0}}\right)^{1 / 2}+d_{2}^{\prime \prime} \frac{E}{c_{0}}+d_{3}^{\prime \prime}\left(\frac{E}{c_{0}}\right)^{3 / 2}+\cdots\right]
$$

where $c_{0}=\left(\partial^{2} V / \partial r^{2}\right)_{o}, d_{1}$ is given by (94) and $d_{2}^{\prime}, d_{3}^{\prime}, \cdots, d_{2}^{\prime \prime}, d_{3}^{\prime \prime}, \cdots$ are respectively minimum and maximum values of certain quantities $d_{2}, d_{3}, \ldots$ which depend on the initial direction of the geodesic along which $r$ is measured and an arbitrary unit vector $\left(\lambda^{i}\right)_{0}$ perpendicular to this direction. All the radial apsides with respect to $O$ which lie on the side of the critical region adjacent to $O$ are minimum apsides, and those on the side remote from $O$ are maximum apsides.

For larger values of $E$, the region $V<E$ may contain critical regions bounded by surfaces of the type (97), (98).

We note that, as $E$ tends to zero, the part of each geodesic from $O$ contained in the critical region limited by (99) and (100) tends to zero as $E^{3 / 2}$. The thickness of the critical region is therefore of the order of $E^{3 / 2}$. On the other hand the critical regions connected with (97) and (98) may have a finite thickness, not tending to zero with $E$.

Let us now consider briefly the case where $O$ is a position of unstable equilibrium, so that for some or all of the geodesics drawn from $O$

$$
\left(\frac{\partial^{2} V}{\partial r^{2}}\right)_{o}<0
$$

We have to consider two cases (i) $E>0$, (ii) $E<0$. 
When $E>0$, we obtain, as before, for those directions at $O$ for which $\left(\partial^{2} V / \partial r^{2}\right)_{o}>0$, a critical region lying between the surfaces (99) and (100) respectively, and also critical regions bounded by (97) and (98), not tending to $O$ as $E$ tends to zero, and therefore excluded from $V<E$ when $E$ is small enough. For those directions for which $\left(\partial^{2} V / \partial r^{2}\right)_{0}<0$, and therefore $c_{0}<0$, no value of $r$ which satisfies (88) will tend to zero as $E$ tends to zero. For geodesics drawn in such directions, the only critical regions will lie between surfaces of the type (97), (98). We have still to consider geodesics for which

$$
\left(\frac{\partial^{2} V}{\partial r^{2}}\right)_{o}=0
$$

which lie on the cone defined by

$$
\left(V_{i j} \mu^{i} \mu^{i}\right)_{o}=0 .
$$

Returning to (86) and (88), we have, connecting $E$ and the distance $r$ to a point at which $\psi_{P}=0$, the equation

$$
E=r^{3}\left(c_{1}+c_{2} r+c_{3} r^{2}+\cdots\right)
$$

where

$$
\begin{aligned}
& c_{1}=\frac{5}{12}\left(\frac{\partial^{3} V}{\partial r^{3}}\right)_{o}, \quad c_{2}=\frac{1}{8}\left(\frac{\partial^{4} V}{\partial r^{4}}\right)_{o}, \\
& c_{3}=\frac{7}{240}\left(\frac{\partial^{5} V}{\partial r^{5}}\right)_{o}+\frac{1}{12}\left(\frac{\partial^{3} V}{\partial r^{3}} K(\lambda, \mu)\right)_{o}
\end{aligned}
$$

Let us leave out of consideration directions satisfying simultaneously (102) and

$$
\left(\frac{\partial^{3} V}{\partial r^{3}}\right)_{0}=0
$$

If

$$
\left(\frac{\partial^{3} V}{\partial r^{3}}\right)_{o}>0, \quad\left(\frac{\partial^{2} V}{\partial r^{2}}\right)_{o}=0, \quad E>0,
$$

then (104) can be reverted to give $r$ in the form

$$
r=\left(\frac{E}{c_{1}}\right)^{1 / 3}\left[1+f_{1}\left(\frac{E}{c_{1}}\right)^{1 / 3}+f_{2}\left(\frac{E}{c_{1}}\right)^{2 / 3}+\cdots\right]
$$

there may also be points which do not tend to $O$ as $E$ tends to zero, given by an equation of the form

$$
r=r_{1}+g_{1} E+g_{2} E^{2}+\cdots .
$$


where $r_{1}$ is such that

$$
b_{3}+b_{4} r_{1}+b_{5} r_{1}^{2}+\cdots=0,
$$

and $r_{1}, g_{1}, g_{2}, \cdots$ depend only on the initial direction of the geodesic at $O$ and on $\left(\lambda^{i}\right)_{o}$. On the other hand, if

$$
\left(\frac{\partial^{3} V}{\partial r^{3}}\right)_{o}<0, \quad\left(\frac{\partial^{2} V}{\partial r^{2}}\right)_{o}=0, \quad E>0,
$$

there is (since $r>0$ ) no reversion of (104) which tends to zero with $E$.

We have still to consider the case where $E<0$. Turning to (88), we see at once that if $\left(\partial^{2} V / \partial r^{2}\right)_{o}>0$, then there is no critical region which tends to $O$ as $E$ tends to zero. ${ }^{*}$ The critical region for such directions will lie between (97) and (98). If $\left(\partial^{2} V / \partial r^{2}\right)_{o}<0$, then there is a critical region which tends to $O$ as $E$ tends to zero, and it lies between the surfaces (99) and (100). The cases where $\left(\partial^{2} V / \partial r^{2}\right)_{O}=0$ may be dealt with in the same manner as before.

Defining an adjacent critical region as one which tends to $O$ as $E$ tends to zero, and a remote critical region as one which does not tend to $O$ as $E$ tends to zero, we may state the following result:

THEOREM VIII. When a system moves with total energy $E$ in the neighborhood of a position of unstable equilibrium $O(V=0)$, then

(i) there exists an adjacent critical region in those directions for which $E\left(\partial^{2} V / \partial r^{2}\right)_{o}>0$ and in those directions for which $\left(\partial^{2} V / \partial r^{2}\right)_{o}=0, E\left(\partial^{3} V / \partial r^{3}\right)_{o}$ $>0$, but not in those directions for which $E\left(\partial^{2} V / \partial r^{2}\right)_{o}<0$ nor in those for which $\left(\partial^{2} V / \partial r^{2}\right)_{o}=0, E\left(\partial^{3} V / \partial r^{3}\right)_{o}<0$;

(ii) in those directions for which an adjacent critical region exists, it lies between the surfaces (99) and (100) if $\left(\partial^{2} V / \partial r^{2}\right)_{O} \neq 0$, and, if $\left(\partial^{2} V / \partial r^{2}\right)_{O}=0$, between the surfaces

$$
\begin{aligned}
& r=\left(\frac{E}{c_{1}}\right)^{1 / 3}\left[1+f_{1}^{\prime}\left(\frac{E}{c_{1}}\right)^{1 / 3}+f_{2}^{\prime}\left(\frac{E}{c_{1}}\right)^{2 / 3}+\cdots\right], \\
& r=\left(\frac{E}{c_{1}}\right)^{1 / 3}\left[1+f_{1}^{\prime \prime}\left(\frac{E}{c_{1}}\right)^{1 / 3}+f_{2}^{\prime \prime}\left(\frac{E}{c_{2}}\right)^{2 / 3}+\cdots\right],
\end{aligned}
$$

where $c_{1}$ is given in (105) and $f_{1}^{\prime}, f_{2}^{\prime}, \cdots, f_{1}^{\prime \prime}, f_{2}^{\prime \prime}, \cdots$ are respectively minimum and maximum values of the coefficients $f_{1}, f_{2}, \cdots$ in (108);

(iii) for directions for which $\left(\partial^{2} V / \partial r^{2}\right)_{o} \neq 0$, remote critical regions correspond to solutions of (95), and lie between the surfaces (97), (98). For directions for which $\left(\partial^{2} V / \partial r^{2}\right)_{o}=0,\left(\partial^{3} V / \partial r^{3}\right)_{o} \neq 0$, remote critical regions correspond to solutions of (110), and lie between surfaces similarly obtained.

* Since $E<0$ and $E-V>0, V$ must be negative. Hence motion is impossible in the region near $O$ reached by geodesics for which $\left(\partial^{2} V / \partial r^{2}\right)_{O}>0$. 
10. Radial-apsides for motion near a pole of the potential energy. Let us investigate the radial-apsides in the neighborhood of $O$, which is a pole of $V$. We shall assume that, in some conical region $R$, having its vertex at $O, V$ may be written

$$
V=\frac{U}{r^{m}} \quad(m>0)
$$

where $U$ can be expanded in a power series in $r$ along each radial geodesic in $R$; $U$ will in general be multiple-valued at $O$. (This will be the case in the problem of three bodies, for example, if the region $R$, whose vertex $O$ is at a position of triple collision, excludes all displacements in which two of the bodies move off in coincidence; $U$ is a constant along each radial geodesic and $m=1$ for this case.) We have

$$
\begin{aligned}
\psi_{F} & =2(E-V) F_{i j} \lambda^{i} \lambda^{j}-r \frac{\partial V}{\partial r}, \\
& =2 E F_{i j} \lambda^{i} \lambda^{i}+\frac{\Phi}{r^{m}},
\end{aligned}
$$

where

$$
\Phi=-2 U F_{i j} \lambda^{i} \lambda^{i}-r \frac{\partial U}{\partial r}+m U
$$

Accordingly in $R$

$$
\psi_{F}=E\left(a_{0}+a_{2} r^{2}+a_{3} r^{3}+\cdots\right)+\frac{1}{r^{m}}\left(b_{0}+b_{1} r+b_{2} r^{2}+\cdots\right) .
$$

where the values of $a_{0}, a_{2}, a_{3}, \cdots$ are as given in (85), and

$$
\begin{aligned}
& b_{0}=(m-2)(U)_{o}, \\
& b_{1}=(m-3)\left(\frac{\partial U}{\partial r}\right)_{o} \\
& b_{2}= \frac{m-4}{2}\left(\frac{\partial^{2} U}{\partial r^{2}}\right)_{o}-\left(U F_{\left.i j k_{1} k_{2} \lambda^{i} \lambda^{i} \mu^{k_{1}} \mu^{k_{2}}\right)_{O},}\right. \\
& b_{n}= \frac{m-n-2}{n !}\left(\frac{\partial^{n} U}{\partial r^{n}}\right)_{O}-\frac{2}{n !}\left\{\frac{n(n-1)}{2 !} \frac{\partial^{n-2} U}{\partial r^{n-2}} F_{i j k_{1} k_{2}} \lambda^{i} \lambda^{j} \mu^{k_{1}} \mu^{k_{2}}+\cdots\right. \\
&\left.+n \frac{\partial U}{\partial r} F_{i j k_{1} \cdots k_{n-1}} \lambda^{i} \lambda^{i} \mu^{k_{1}} \cdots \mu^{k_{n-1}}+U F_{i j k_{1} \cdots k_{n}} \lambda^{i} \lambda^{i} \mu^{k_{1}} \cdots \mu^{k_{n}}\right\}_{O} \\
& \quad(n=3,4, \cdots)
\end{aligned}
$$

where $(U)_{o}$ and the derivatives depend on the direction of the radial geodesic at $O$. Here, as before, the vector $\lambda^{i}$ is propagated parallelly along the radial geodesic $O P$, and $\mu^{i}$ is the unit tangent vector to $O P$. 
At any point $P$ the generalized force is

$$
-V^{i}=-\frac{U^{i}}{r^{m}}+\frac{m U \mu^{i}}{r^{m+1}} .
$$

The outward component of force along the geodesic is

$$
-V^{i} \mu_{i}=-\frac{U_{i} \mu^{i}}{r^{m}}+\frac{m U}{r^{m+1}} .
$$

As $r$ tends to zero, the sign of this quantity is that of $(U)_{o}$. Let us confine our attention to attractive fields of force, for which

$$
(U)_{O}<0,
$$

for all directions $\left(\mu^{i}\right)_{o}$ in $R$. Inspection of (118) shows that $m=2$ is an important critical case (as we might have suspected from the results of $\S 7$ ), for the sign of $m-2$ now determines the sign of $b_{0}$.

For a given value of $E$, the sign of $\psi_{F}$ in a region sufficiently close to $O$ (and, of course, belonging to $R$, as is always understood) is that of $b_{0}$. Accordingly we may state the following result:

TheOREM IX. When the potential energy is of the form (114), and the force near $O$ is attractive in a conical region $R$, then for given total energy $E$, all radialapsides near $O$ in $R$ are minimum apsides if $m<2$ and maximum apsides if $m>2$.

If $m=2$, the apsides near $O$ in $R$ are minimum or maximum according as $(\partial U / \partial r)_{o}$ is negative or positive for the direction of the geodesic drawn to the adjacent position in question.

If $\psi_{F}=0$ at a point $P$, then, by (117), we have

$$
-\frac{1}{E}=r^{m} \frac{a_{0}+a_{2} r^{2}+a_{3} r^{3}+\cdots}{b_{0}+b_{1} r+b_{2} r^{2}+\cdots} .
$$

In connection with the series development, the case of principal interest is that in which $E$ is negative and very large. This would arise if the system were to start from rest at a position adjacent to $O$. Since $a_{0}=2$, we can revert (122) to obtain a value of $r$ tending to zero as $E$ tends to $-\infty$ only if the first of the $b$ coefficients is positive. Let us, then, assume that $m<2$, so that $b_{0}>0$. Any critical regions which do not tend to $O$ as $E$ tends to $-\infty$ will in the limit consist of points for which $F_{i j} \lambda^{i} \lambda^{i}=0$, and at which, therefore, the sphere with center $O$ possesses asymptotic directions. Such regions will be contained between surfaces with polar equations of the form 


$$
\begin{gathered}
a_{0}+a_{2}^{\prime} r^{2}+a_{3}^{\prime} r^{3}+\cdots=0 \\
a_{0}+a_{2}^{\prime \prime} r^{2}+a_{3}^{\prime \prime} r^{3}+\cdots=0
\end{gathered}
$$

where $a_{2}^{\prime}, a_{3}^{\prime}, \cdots, a_{2}^{\prime \prime}, a_{3}^{\prime \prime}, \cdots$ represent respectively minimum and maximum values for arbitrary choice of the unit vector $\left(\lambda^{i}\right)_{o}$, perpendicular to $\left(\mu^{i}\right)_{o}$. Such critical regions will lie outside the region of motion $(V<E)$ if $E$ is made sufficiently large and negative.

Let us now consider the critical region which tends to $O$ as $E$ tends to $-\infty$. Equation (122) gives

$$
\left(-\frac{b_{0}}{2 E}\right)^{1 / m}=r\left(1+c_{1} r+c_{2} r^{2}+\cdots\right),
$$

where

$$
\begin{aligned}
c_{1}= & -\frac{b_{1}}{m b_{0}}=-\frac{m-3}{m(m-2)}\left(\frac{1}{U} \frac{\partial U}{\partial r}\right)_{o} \\
c_{2}= & \frac{1}{m}\left(\frac{a_{2}}{2}-\frac{b_{2}}{b_{0}}\right)+\frac{m+1}{2 m^{2}}\left(\frac{b_{1}}{b_{0}}\right)^{2} \\
= & -\frac{K(\lambda, \mu)}{3(m-2)}-\frac{m-4}{2 m(m-2)}\left(\frac{1}{U} \frac{\partial^{2} U}{\partial r^{2}}\right)_{0} \\
& +\frac{(m+1)(m-3)^{2}}{2 m^{2}(m-2)^{2}}\left(\frac{1}{U} \frac{\partial}{\partial r}\right)_{0}^{2} .
\end{aligned}
$$

Accordingly we have, for that value of $r$ which tends to zero as $E$ tends to $-\infty$,

where

$$
\begin{aligned}
r= & \left(-\frac{(m-2)(U)_{o}}{2 E}\right)^{1 / m}\left[1+d_{1}\left(-\frac{(m-2)(U)_{o}}{2 E}\right)^{1 / m}\right. \\
& \left.+d_{2}\left(-\frac{(m-2)(U)_{o}}{2 E}\right)^{2 / m}+\cdots\right]
\end{aligned}
$$

$$
\begin{gathered}
d_{1}=-c_{1}=\frac{m-3}{m(m-2)}\left(\frac{1}{U} \frac{\partial U}{\partial r}\right)_{o} \\
d_{2}=2 c_{1}^{2}-c_{2}=\frac{K(\lambda, \mu)}{3(m-2)}+\frac{m-4}{2 m(m-2)}\left(\frac{1}{U} \frac{\partial^{2} U}{\partial r^{2}}\right)_{0} \\
\quad-\frac{(m-3)^{3}}{2 m^{2}(m-2)^{2}}\left(\frac{1}{U} \frac{\partial U}{\partial r}\right)_{0}^{2}
\end{gathered}
$$


We may state the following result:

THEOREM X. When the potential energy is of the form (114), and the force near $O$ is attractive, there will exist a critical region which tends to $O$ as $E$ tends to $-\infty$ if $m<2$. Such critical regions are contained between surfaces whose polar equations are of the form (127), the quantities $d_{2}, d_{3}, \cdots$ being replaced first by their minimum values (for arbitrary unit vectors $\lambda^{i}$ at $O$, perpendicular to the direction of the geodesic along which $r$ is measured) and then by their maximum values. The thickness of the critical region is of the order $|E|^{-3 / m}$.

If $m=2$, the existence of a critical region, tending to $O$ as $E$ tends to $-\infty$, demands that $b_{1}$ be positive, $b_{0}$ being zero if $m=2$. Instead of (125), we shall now have an equation of the form

$$
-\frac{b_{1}}{2 E}=r\left(1+e_{1} r+e_{2} r^{2}+\cdots\right)
$$

from which we obtain, instead of (127), an equation of the form

$$
r=\frac{1}{2 E}\left(\frac{\partial U}{\partial r}\right)_{o}\left[1+f_{1} \frac{1}{2 E}\left(\frac{\partial U}{\partial r}\right)_{o}+f_{2}\left(\frac{1}{2 E}\left(\frac{\partial U}{\partial r}\right)_{o}\right)^{2}+\cdots\right]
$$

\section{Part III. Potential-APsides}

11. Potential-apsides in general. For the consideration of potential-apsides, we have, by (16) and (15),

$$
\begin{aligned}
\frac{d^{2} V}{d t^{2}} & =\psi_{V}(x, \lambda, E), \\
\psi_{V}(x, \lambda, E) & =2(E-V) V_{i j} \lambda^{i} \lambda^{i}-V_{i} V^{i} .
\end{aligned}
$$

Let $O$ be an ordinary point, with respect to $V$, for a motion with total energy $E$; that is, it is not a position of instantaneous rest, nor a position of equilibrium, nor a pole of $V$. Then if $\lambda^{i}$ is tangent to $V=$ const., we have, by (25),

$$
V_{i j} \lambda^{i} \lambda^{i}=k(V, \lambda)\left(V_{i} V^{i}\right)^{1 / 2}
$$

where $k(V, \lambda)$ is the curvature of the equipotential surface for the direction $\lambda^{i}$, positive when the surface is concave on the side from which the line of force $\left(-V^{i}\right)$ proceeds. But

$$
\left(V_{i} V^{i}\right)^{1 / 2}=X,
$$

the magnitude of the force; therefore

$$
\psi_{V}(x, \lambda, E)=X\{2(E-V) k(V, \lambda)-X\} .
$$


Accordingly we may state the following result:

THEOREM XI. For motion with total energy $E$, an ordinary point belongs to the region of minimum potential-apsides if the curvature of the equipotential surface at the point (defined as in \$4) is greater than

$$
\frac{X}{2(E-V)}
$$

for every direction in the surface, $X$ being the magnitude of the force; and the point belongs to the region of maximum potential-apsides if the curvature is less than this quantity for every direction in the surface. Every point at which the equipotential surface is convex on the side from which the line of force issues belongs to the region of maximum potential-apsides.*

12. Potential-apsides for flat manifolds with homogeneous potential energy. Let us now suppose that the manifold is flat, and that, in terms of the normal coördinates for which the kinetic energy is of the form (56), the potential energy $V$ is homogeneous of degree $n$ in the coördinates. For these coördinates covariant differentiation reduces to ordinary differentiation. Then $V_{i}$ is homogeneous of degree $n-1$, and $V_{i j}$ is homogeneous of degree $n-2$. As we proceed along a radial geodesic from $O$, we have therefore

$$
V=\left(\frac{r}{r_{1}}\right)^{n}(V)_{1}, V_{i}=\left(\frac{r}{r_{1}}\right)^{n-1}\left(V_{i}\right)_{1}, V_{i j}=\left(\frac{r}{r_{1}}\right)^{n-2}\left(V_{i j}\right)_{1},
$$

where the subscript (1) denotes evaluation at the point where the radial geodesic cuts the sphere $r=r_{1}$. Thus, by (132), for any point on this geodesic,

$$
\psi_{V}(x, \lambda, E)=\left(\frac{r}{r_{1}}\right)^{n-2}\left\{2 E\left(V_{i j} \lambda^{i} \lambda^{i}\right)_{1}-\left(\frac{r}{r_{1}}\right)^{n}\left(2 V \cdot V_{i j} \lambda^{i} \lambda^{i}+: V_{i} V^{i}\right)_{1}\right\},
$$

where the values of $\lambda^{i}$ at $r=r_{1}$ are obtained by parallel propagation, $\lambda^{i}$ being (since the manifold is flat) constants along the geodesic. Thus, as $r$ ranges from 0 to $\infty, \psi_{V}$ vanishes (for assigned $\lambda^{i}$ ) at most once, and that for $r$ satisfying

$$
\left(\frac{r}{r_{1}}\right)^{n}=\frac{2 E\left(V_{i j} \lambda^{i} \lambda^{j}\right)_{1}}{\left(2 V \cdot V_{i j} \lambda^{i} \lambda^{j}+V_{i} V^{i}\right)_{1}}
$$

or, by (133) and (134), if $\lambda^{i}$ is tangential to the equipotential surfaces,

$$
\left(\frac{r_{1}}{r}\right)^{n}=\frac{(V)_{1}}{E}+\frac{(X)_{1}}{2 E(k(V, \lambda))_{i}}
$$

* Cf. Hadamard, loc. cit., p. 360. 
where $(X)_{1}$ is the intensity and $(k(V, \lambda))_{1}$ the curvature of the equipotential surface at $r=r_{1}$ on the geodesic, counted positive if the surface is concave on the side from which the line of force issues.

If the equipotential surface at $r=r_{1}$ on the geodesic has a single-signed curvature for all tangential directions $\lambda^{i}$, then (140) determines a region on the radial geodesic belonging to the critical region; it is bounded by the two points corresponding to the values of $r$ obtained from (140) when we substitute in turn for $(k(V, \lambda))_{1}$ its greatest and least values.

We may express our results in a slightly simpler form if the values of $E$ are such that there exists a surface of instantaneous rest $V=E$. We shall then take $r_{1}$ at the intersection of the radial geodesic with this surface, and employ a subscript $(E)$ to denote evaluation at this point. Equation (140) then becomes

$$
\left(\frac{r_{E}}{r}\right)^{n}=1+\frac{(X)_{E}}{2 E(k(V, \lambda))_{E}}, \text { or } \frac{E}{V}=1+\frac{(X)_{E}}{2 E(k(V, \lambda))_{E}}
$$

We may state our result as follows:

THEOREM XII. When the manifold of configurations is flat and the potential energy is homogeneous of degree $n$ in the normal coordinates, any radial geodesic drawn from the origin has at most one segment contained in the critical region with respect to potential-apsides; the other two parts of the geodesic belong one to the region of minimum apsides and the other to the region of maximum apsides. The bounding surfaces of the critical region are given by (140) in the manner described above. If the total energy is such that the surface of instantaneous rest $(V=E)$ exists, the bounding surfaces of the critical region are given by the polar equations

$$
\begin{aligned}
& r=r_{E}\left\{1+\frac{(X)_{E}}{2 E k_{E}^{\prime}}\right\}^{-1 / n}, \\
& r=r_{E}\left\{1+\frac{(X)_{E}}{2 E k_{E}^{\prime \prime}}\right\}^{-1 / n},
\end{aligned}
$$

where $r_{E}$ is the distance from $O$ to the surface $V=E$, along the direction of the radial geodesic along which $r$ is measured, $(X)_{E}$ is the intensity at the point where this geodesic cuts $V=E$ and $k_{E}^{\prime}, k_{E}^{\prime \prime}$ are respectively the least and greatest curvatures (with signs attributed according to the convention described above) of the surface $V=E$, for arbitrary tangential directions.

To determine whether the region adjacent to $O$ (on an assigned geodesic) belongs to the region of minimum or maximum apsides, we have to examine 
the sign of $\psi_{V}$ (as given by (138)) as $r$ tends to zero. We may write (138) in the form

$$
\psi_{V}(x, \lambda, E)=(X)_{1}\left(\frac{r}{r_{1}}\right)^{n-2}\left\{2 E(k(V, \lambda))_{1}-\left(\frac{r}{r_{1}}\right)^{n}(X+2 V k(V, \lambda))_{1}\right\} .
$$

As $r$ tends to zero, the first or the second term dominates according as $n$ is positive or negative. We shall leave out of consideration the case $n=0$. The following results are then immediate:

THEOREM XIII. When the manifold of configurations is flat and the potential energy is homogeneous of degree $n$ in normal coordinates, the potential-apsides in the part of a conical region $R$ adjacent to the origin are minimum apsides, under any of the following circumstances:

(i) $n>0, E>0$; in $R$ the equipotential surfaces have positive curvature (i.e. are concave on the side from which the line of forces issues); or

(ii) $n>0, E<0$; in $R$ the equipotential surfaces have negative curvature (i.e. are convex on the side from which the line of force issues); or

(iii) $n<0$; in $R$ the value of $-2 V k(V, \lambda)$ exceeds the intensity $X$, for all directions $\lambda^{i}$ tangential to the equipotential surfaces.

The pctential-apsides in $R$ are maximum apsides under the following circumstances:

(i) $n>0, E>0$; in $R$ the equipotential surfaces have negative curvature;

(ii) $n>0, E<0$; in $R$ the equipotential surfaces have positive curvature;

(iii) $n<0$; in $R$ the value of $-2 V k(V, \lambda)$ is less than the intensity $X$, for all directions $\lambda^{i}$ tangential to the equipotential surfaces.

It is clear that the critical region reduces to a surface when the number of degrees of freedom of the system is two, or when the equipotential surfaces are spheres. It is interesting to investigate the significance of the above results in the case of a particle performing finite stable oscillations in a plane, the potential energy being

$$
V=\frac{1}{2}\left(\frac{x^{2}}{a^{2}}+\frac{y^{2}}{b^{2}}\right)
$$

Here $n=2$, and the critical curve, separating the region of minimum apsides from that of maximum apsides, has the polar equation (141) or

$$
\left(\frac{r_{E}}{r}\right)^{2}=1+\frac{(X)_{E}}{2 E k_{E}}
$$

where $k_{E}$ is the curvature of the equipotential curve $V=E$, or

$$
\frac{x^{2}}{a^{2}}+\frac{y^{2}}{b^{2}}=2 E
$$


at the point $Q$ where it is met by the radius vector along which $r$ is measured; $k_{E}$ will be positive. We have

$$
k_{E}=\frac{1}{4 E^{2}} \frac{p^{3}}{a^{2} b^{2}}, \quad(X)_{E}=\frac{2 E}{p},
$$

where $p$ is the perpendicular from $O$ on the tangent at $Q$ to the ellipse (146); thus (145) gives

$$
\left(\frac{r_{E}}{r}\right)^{2}=1+\frac{4 E^{2} a^{2} b^{2}}{p^{4}}, \quad r=\frac{r_{E} p^{2}}{\left(p^{4}+4 E^{2} a^{2} b^{2}\right)^{1 / 2}}
$$

as the polar equation of the critical curve; in parametric form the curve is

$$
\begin{aligned}
& x=\frac{a^{2} b(2 E)^{1 / 2} \cos \theta}{\left(a^{2} b^{2}+\left(b^{2} \cos ^{2} \theta+a^{2} \sin ^{2} \theta\right)^{2}\right)^{1 / 2}}, \\
& y=\frac{a b^{2}(2 E)^{1 / 2} \sin \theta}{\left(a^{2} b^{2}+\left(b^{2} \cos ^{2} \theta+a^{2} \sin ^{2} \theta\right)^{2}\right)^{1 / 2}} .
\end{aligned}
$$

We note that when $b=a$, so that the equipotentials are circles, and potentialapsides become confounded with radial-apsides, we have for the critical circle

$$
r=r_{E} / 2^{1 / 2},
$$

agreeing with the result of Theorem IV.

13. Potential-apsides near a surface of instantaneous rest. For motion with total energy $E$ the surface

$$
V=E
$$

is a surface of instantaneous rest. The $V$-apsidal function at a point on this surface is, by (132),

$$
\psi_{V}=-V_{i} V^{i}<0
$$

Hence we have the result

THEOREM XIV. All potential-apsides near a surface of instantaneous rest are maximum apsides.

14. Potential-apsides near a position of equilibrium. Let $O$ be a position of equilibrium, for which we choose $V=0$. We have then

$$
(V)_{o}=0,\left(V_{i}\right)_{o}=0 \text {. }
$$

Let us take any point $P$, with which there is associated a unit vector $\lambda^{i}$. Let us draw the geodesic $O P$ (of length $r$ ), and let $\mu^{i}$ be the unit vector tangent to this geodesic. Then, defining $\lambda^{i}$ along $O P$ by parallel propagation, we may develop $\psi_{V}$ (given by (132)) as a power series in $r$. Thus we obtain 


$$
\psi_{V}=E\left(a_{0}+a_{1} r+a_{2} r^{2}+\cdots\right)+b_{2} r^{2}+b_{3} r^{3}+\cdots,
$$

where

$$
\begin{aligned}
& a_{0}=2\left(V_{i j} \lambda^{i} \lambda^{j}\right)_{O}, \\
& a_{1}=2\left(V_{i j k_{1}} \lambda^{i} \lambda^{j} \mu^{k_{1}}\right)_{o} \text {, } \\
& a_{n}=\frac{2}{n !}\left(V_{i j k_{1} \cdots k_{n}} \lambda^{i} \lambda^{i} \mu^{k_{1}} \cdots \mu^{k_{n}}\right)_{o} \quad \quad(n=2,3, \cdots) \text {; } \\
& b_{2_{-}}=-\left(V_{i j} \lambda^{i} \lambda^{j}\right)_{o}\left(V_{k l} \mu^{k} \mu^{l}\right)_{o}-\left(V_{i j} \mu^{j} V^{i k} \mu_{k}\right)_{o},
\end{aligned}
$$

$$
\begin{aligned}
b_{n}= & -\frac{2}{n !}\left(V_{i j} \lambda^{i} \lambda^{i} V_{k_{1} \cdots k_{n}} \mu^{k_{1}} \cdots \mu^{k_{n}}+n V_{i j k_{1}} \lambda^{i} \lambda^{i} \mu^{k_{1}} V_{k_{2} \cdots k_{n}} \mu^{k_{2}} \cdots \mu^{k_{n}}+\cdots\right. \\
& \left.+\frac{n(n-1)}{2 !} V_{i j k_{1} \cdots k_{n-2} \lambda^{i} \lambda^{i} \mu^{k_{1}} \cdots \mu^{k_{n-2}} V_{k_{n-1} k_{n}} \mu^{k_{n-1}} \mu^{k_{n}}}\right)_{O} \\
& -\frac{1}{n !}\left(n V_{i k_{1} \mu^{k_{1}} V^{i k_{2} \cdots k_{n}} \mu_{k_{2}} \cdots \mu_{k_{n}}+\cdots}\right. \\
& +n V_{\left.i k_{1} \cdots k_{n-1} \mu^{k_{1}} \cdots \mu^{k_{n-1}} V^{i k_{n}} \mu_{k_{n}}\right)_{O}} \quad(n=3,4, \cdots) .
\end{aligned}
$$

We observe that if $V$ is a minimum at $\delta, a_{0}$ is positive for arbitrary $\left(\lambda^{i}\right)_{0}$; moreover in this case $E$ is necessarily positive. On the other hand, if $V$ is a maximum at $O, a_{0}$ is negative, but $E$ may be positive or negative. Hence we may state the following result:

THEOREM XV. In the immediate neighborhood of a position of stable equilibrium, all the potential-apsides are minimum apsides. In the immediate neighborhood of a position of equilibrium for which $V$ is a maximum, all the potentialapsides are maximum apsides if $E$ is positive.

We have not included in this theorem a statement for the case where $V$ is a maximum and $E$ is negative, because we are at present only concerned with the state of affairs when $r$ tends to zero, $E$ being fixed. Under the above circumstances, the region for which $r$ tends to zero (the immediate neighborhood of $O$ ) lies in the region for which $E-V<0$, and is therefore forbidden to the system.

When we employ the function $\psi_{V}$ to determine the regions of minimum and maximum apsides, we are only interested in those vectors $\lambda^{i}$ which are tangential to the equipotential surfaces. The preceding method for the calculation of $\psi_{V}$ (in which $\lambda^{i}$ is propagated parallelly along the radial geodesic) does not lend itself to the realisation of this condition. We shall therefore in- 
troduce another law of propagation of $\lambda^{i}$, which will ensure the satisfaction of the condition

$$
V_{i} \lambda^{i}=0,
$$

at all points of the radial geodesic.

Let us draw another radial geodesic, adjacent to $O P$, and let $\eta^{i}$ be the infinitesimal displacement-vector from a point on $O P$ to the point on the other geodesic where $V$ has the same value. The relation

$$
V_{i} \eta^{i}=0
$$

is then satisfied along $O P$. The equation of geodesic deviation is*

$$
\frac{\delta^{2} \eta^{i}}{\delta r^{2}}+R_{\cdot j k l}^{i} \mu^{i} \eta^{k} \mu^{l}-\theta \mu^{i}=0,
$$

where $\theta$ depends on the nature of the correspondence between the points of the two geodesics. From (157) we derive

$$
\begin{aligned}
& V_{i} \frac{\delta \eta^{i}}{\delta r}+V_{i j} \eta^{i} \mu^{j}=0 \\
& V_{i} \frac{\delta^{2} \eta^{i}}{\delta r^{2}}+2 V_{i j} \frac{\delta \eta^{i}}{\delta r} \mu^{j}+V_{i j k} \eta^{i} \mu^{i} \mu^{k}=0
\end{aligned}
$$

and hence, multiplying (158) by $V_{i}$, we have

$$
\theta \cdot V_{i} \mu^{i}=R_{\cdot j k l}^{i} V_{i} \mu^{i} \eta^{k} \mu^{l}-2 V_{i j} \frac{\delta \eta^{i}}{\delta r} \mu^{j}-V_{i j k} \eta^{i} \mu^{j} \mu^{k},
$$

where

$$
V_{i} \mu^{i}=\frac{\partial V}{\partial r}
$$

Let us define the unit vector $\lambda^{i}$ by

$$
\lambda^{i}=\eta^{i} / \eta \quad\left(\eta^{2}=\eta_{i} \eta^{i}\right)
$$

$\lambda^{i}$ satisfies (156) along OP. Differentiating (156), we have

$$
V_{i} \frac{\delta \lambda^{i}}{\delta r}+V_{i j} \lambda^{i} \mu^{i}=0
$$

* Cf. Proceedings of the Royal Irish Academy, vol. 39A (1929), p. 14, for a simple derivation of the formula, first obtained (for a general correspondence) by Levi-Civita, Mathematische Annalen, vol. 97 (1926), p. 315. 
and therefore at $O$, since $V_{i}=0$ there,

$$
\left(V_{i j} \lambda^{i} \mu^{j}\right)_{O}=0 .
$$

Further, it is evident that the vectors $\left(\delta \eta^{i} / \delta r\right)_{o}$ and $\left(\lambda^{i}\right)_{o}$ are codirectional, so that we may put

$$
\left(\frac{\delta \eta^{i}}{\delta r}\right)_{o}=\epsilon\left(\lambda^{i}\right)_{o}
$$

where $\epsilon$ is a constant which tends to zero as the neighboring geodesic tends to coincidence with $O P$.

Equations (158) (with substitution for $\theta$ from (161)), together with the boundary conditions (166) and

$$
\left(\eta^{i}\right)_{o}=0,
$$

determine the infinitesimal displacement vector $\eta^{i}$ along $O P$, and hence the unit vector $\lambda^{i}$, the initial value $\left(\lambda^{i}\right)_{o}$ satisfying (165).

Now we have

$$
\psi_{V}=\frac{2(E-V) V_{i j} \eta^{i} \eta^{j}}{\eta^{2}}-V_{i} V^{i} .
$$

The various terms occurring may be expanded in power series in $r$. As the calculation soon becomes intricate, we shall only calculate a few terms explicitly.

Differentiating (161), we have

$$
\begin{aligned}
\frac{\partial \theta}{\partial r} \frac{\partial V}{\partial r}+\theta \frac{\partial^{2} V}{\partial r^{2}}= & R_{\cdot j k l m}^{i} V_{i} \mu^{j} \eta^{k} \mu^{l} \mu^{m}+R_{\cdot j k l}^{i} V_{i m} \mu^{j} \eta^{k} \mu^{l} \mu^{m} \\
& +R_{\cdot j k l}^{i} V_{i} \mu^{j} \frac{\delta \eta^{k}}{\delta r} \mu^{l}-2 V_{i j k} \frac{\delta \eta^{i}}{\delta r} \mu^{i} \mu^{k}-2 V_{i j} \frac{\delta^{2} \eta^{i}}{\delta r^{2}} \mu^{j} \\
& -V_{i j k l} \eta^{i} \mu^{j} \mu^{k} \mu^{l}-V_{i j k} \frac{\delta \eta^{i}}{\delta r} \mu^{j} \mu^{k}
\end{aligned}
$$

which gives, on putting $r=0$, and using (153), (158) and (166),

$$
\begin{aligned}
(\theta)_{o}\left(\frac{\partial^{2} V}{\partial r^{2}}\right)_{o} & =-2 \epsilon\left(V_{i j k} \lambda^{i} \mu^{j} \mu^{k}\right)_{o}-2\left(V_{i j} \mu^{i} \mu^{j} \theta\right)_{o}-\epsilon\left(V_{i j k} \lambda^{i} \mu^{i} \mu^{k}\right)_{o} \\
(\theta)_{o} & =-\epsilon\left(V_{i j k} \lambda^{i} \mu^{i} \mu^{k}\right)_{o} /\left(\partial^{2} V / \partial r^{2}\right)_{o} \quad\left(\partial^{2} V / \partial r^{2}=V_{i j} \mu^{i} \mu^{j}\right) .
\end{aligned}
$$

Differentiating (169), and putting $r=0$, we obtain

$$
\begin{aligned}
4\left(\frac{\partial \theta}{\partial r}\right)_{o}\left(\frac{\partial^{2} V}{\partial r^{2}}\right)_{o}+6(\theta)_{o}\left(\frac{\partial^{3} V}{\partial r^{3}}\right)_{o} & =4 \epsilon\left(R_{\cdot j k l}^{i} V_{i m} \mu^{j} \lambda^{k} \mu^{l} \mu^{m}\right)_{o} \\
& -4 \epsilon\left(V_{i j k l} \lambda^{i} \mu^{j} \mu^{k} \mu^{l}\right)_{o}
\end{aligned}
$$


or

$$
\left(\frac{\partial \theta}{\partial r}\right)_{o}\left(\frac{\partial^{2} V}{\partial r^{2}}\right)_{o}^{2}=\frac{3 \epsilon}{2}\left(V_{i j k} \lambda^{i} \mu^{j} \mu^{k}\right)_{o}\left(\frac{\partial^{3} V}{\partial r^{3}}\right)_{o}+\epsilon\left(R_{\cdot j k l}^{i} V_{i m} \mu^{\lambda} \lambda^{k} \mu^{l} \mu^{m}\right)_{o}\left(\frac{\partial^{2} V}{\partial r^{2}}\right)_{o}
$$

Therefore we have

$$
-\epsilon\left(V_{i j k l} \lambda^{i} \mu^{j} \mu^{k} \mu^{l}\right)_{o}\left(\frac{\partial^{2} V}{\partial r^{2}}\right)_{o} .
$$

$$
\theta=\epsilon\left(a_{0}+a_{1} r+a_{2} r^{2}+\cdots\right),
$$

where

$$
\begin{aligned}
a_{0}= & -\left(\frac{V_{i j k} \lambda^{i} \mu^{i} \mu^{k}}{\partial^{2} V / \partial r^{2}}\right)_{o} \\
a_{1}= & \frac{3}{2}\left(\frac{V_{i j k} \lambda^{i} \mu^{i} \mu^{k}\left(\partial^{3} V / \partial r^{3}\right)}{\left(\partial^{2} V / \partial r^{2}\right)^{2}}\right)_{o}+\left(\frac{R_{\cdot j k l}^{i} V_{i m} \mu^{i} \lambda^{k} \mu^{l} \mu^{m}}{\partial^{2} V / \partial r^{2}}\right)_{o} \\
& -\left(\frac{V_{i j k l} \lambda^{i} \mu^{i} \mu^{k} \mu^{l}}{\partial^{2} V / \partial r^{2}}\right)_{o} .
\end{aligned}
$$

Let us now expand $\eta^{2}$; we have

$$
\eta^{2}=\eta_{i} \eta^{i}=\epsilon^{2}\left(b_{2} r^{2}+b_{3} r^{3}+b_{4} r^{4}+\cdots\right),
$$

where

$$
\begin{aligned}
b_{2} & =\frac{1}{\epsilon^{2}}\left(\frac{\delta \eta_{i}}{\delta r} \frac{\delta \eta^{i}}{\delta r}\right)_{o}=1, \\
b_{3} & =\frac{1}{\epsilon^{2}}\left(\frac{\delta \eta_{i}}{\delta r} \frac{\delta^{2} \eta^{i}}{\delta r^{2}}\right)_{o}=\frac{1}{\epsilon}\left(\lambda_{i} \mu^{i} \theta\right)_{o}=\left(\lambda_{i} \mu^{i}\right)_{o} a_{0}=-\left(\lambda_{m} \mu^{m}\right)_{o}\left(\frac{V_{i j k} \lambda^{i} \mu^{j} \mu^{k}}{\partial^{2} V / \partial r^{2}}\right)_{O} \\
b_{4} & =\frac{1}{\epsilon^{2}}\left(\frac{1}{3} \frac{\delta \eta_{i}}{\delta r} \frac{\delta^{3} \eta^{i}}{\delta r^{3}}+\frac{1}{4} \frac{\delta^{2} \eta_{i}}{\delta r^{2}} \frac{\delta^{2} \eta^{i}}{\delta r^{2}}\right)_{o} \\
& =\frac{1}{3}\left(\lambda_{i} \mu^{i}\right)_{o} a_{1}-\frac{1}{3}\left(R_{i j k l} \lambda^{i} \mu^{3} \lambda^{k} \mu^{l}\right)_{o}+\frac{1}{4} a_{0}{ }^{2} .
\end{aligned}
$$

Also

$$
V_{i j} \eta^{i} \eta^{j}=\epsilon^{2}\left(c_{2} r^{2}+c_{3} r^{3}+\cdots\right)
$$

where, by virtue of (165),

$$
\begin{aligned}
& c_{2}=\frac{1}{\epsilon^{2}}\left(V_{i j} \frac{\delta \eta^{i}}{\delta r} \frac{\delta \eta^{j}}{\delta r}\right)_{O}=\left(V_{i j} \lambda^{i} \lambda^{j}\right)_{o}, \\
& c_{3}=\frac{1}{\epsilon^{2}}\left(V_{i j k} \frac{\delta \eta^{i}}{\delta r} \frac{\delta \eta^{j}}{\delta r} \mu^{k}+V_{i j} \frac{\delta \eta^{i}}{\delta r} \frac{\delta^{2} \eta^{j}}{\delta r^{2}}\right)_{O}=\left(V_{i j k} \lambda^{i} \lambda^{j} \mu^{k}\right)_{O}, \\
& c_{4}=\frac{1}{2}\left(V_{i j k l} \lambda^{i} \lambda^{j} \mu^{k} \mu^{l}\right)_{O}-\frac{3}{4} a_{0}\left(V_{i j k} \lambda^{i} \mu^{j} \mu^{k}\right)_{O}-\frac{1}{3}\left(R_{\cdot j k l}^{i} V_{i m} \mu^{i} \lambda^{k} \mu^{l} \mu^{m}\right)_{O},
\end{aligned}
$$


and

$$
V=d_{2} r^{2}+d_{3} r^{3}+\cdots
$$

where

$$
d_{2}=\frac{1}{2}\left(\partial^{2} V / \partial r^{2}\right)_{o}, d_{3}=\frac{1}{6}\left(\partial^{3} V / \partial r^{3}\right)_{o}
$$

and

$$
V_{i} V^{i}=e_{2} r^{2}+e_{3} r^{3}+\cdots,
$$

where

$$
\begin{aligned}
& e_{2}=\left(V_{i j} \mu^{j} V^{i k} \mu_{k}\right)_{O} \\
& e_{3}=\left(V_{i j k} \mu^{i} \mu^{k} V^{i l} \mu_{l}\right)_{O}
\end{aligned}
$$

Substituting the series in (168), we have

$$
\psi_{V}=2 E \frac{c_{2}+c_{3} r+\cdots}{1+b_{3} r+\cdots}
$$

$$
-r^{2}\left[e_{2}+e_{3} r+\cdots+2\left(d_{2}+d_{3} r+\cdots\right) \frac{c_{2}+c_{3} r+\cdots}{1+b_{3} r+\cdots}\right] .
$$

In this expression, the $b$ 's and $c^{\prime}$ 's depend on $\left(\lambda^{i}\right)_{o}$, but the $d$ 's and $e^{\text {'s do not. }}$

Let us confine our attention to the case where $O$ is a position of stable equilibrium, so that $V$ is a minimum at $O\left(\left(\partial^{2} V / \partial r^{2}\right)_{o}>0\right)$, and $E>0$.

The points on $O P$ for which $\psi_{V}=0$, for assigned $\left(\lambda^{i}\right)_{o}$ satisfying (165), will, in general, consist of those points which tend to positions other than $O$ as $E$ tends to zero, and that point which tends to zero as $E$ tends to zero. The limiting positions of the former are given by equating to zero the bracket [ ] in (183); the position of the latter is given in terms of $E$ by a series of the form

$$
r=E^{1 / 2}\left(f_{0}+f_{1} E^{1 / 2}+f_{2} E+\cdots\right)
$$

in which $f_{0}, f_{1}, \cdots$ are functions of $\left(\lambda^{i}\right)_{o}$, to be determined in terms of the coefficients in (183) by the equation

$$
\begin{aligned}
2 E\left(c_{2}+c_{3} r\right. & \left.+c_{4} r^{2}+\cdots\right) \\
& =r^{2}\left[\left(1+b_{3} r+\cdots\right)\left(e_{2}+e_{3} r+\cdots\right)\right. \\
& \left.+2\left(c_{2}+c_{3} r+\cdots\right)\left(d_{2}+d_{3} r+\cdots\right)\right] .
\end{aligned}
$$


We find

$$
\begin{aligned}
& f_{0}=\left(\frac{2 c_{2}}{e_{2}+2 c_{2} d_{2}}\right)^{1 / 2}=\left(\frac{2\left(V_{i j} \lambda^{i} \lambda^{j}\right)_{o}}{\left(V_{i j} \mu^{j} V^{i k} \mu_{k}\right)_{o}+\left(V_{i j} \lambda^{i} \lambda^{j}\right)_{o}\left(\partial^{2} V / \partial r^{2}\right)_{o}}\right)^{1 / 2}, \\
& f_{1}=\frac{f_{0}^{2}}{2\left(e_{2}+2 c_{2} d_{2}\right)}\left\{\frac{2 c_{3}}{f_{0}^{2}}-2\left(c_{2} d_{3}+c_{3} d_{2}\right)-\left(b_{3} e_{2}+e_{3}\right)\right\} .
\end{aligned}
$$

We see that $f_{0}$ and $f_{1}$ do not involve the curvature tensor; $f_{2}$ will involve it, through $b_{4}$ and $c_{4}$. We are to remember that, by carrying out the process given, it is possible to compute as many of the $f$ 's as we please as explicit functions of $\left(\lambda^{i}\right)_{o},\left(\mu^{i}\right)_{o}$, the covariant derivatives of $V$ at $O$, and the curvature tensor and its covariant derivatives at $O$. To find the range of the critical region on the radial geodesic $O P$, defined by $\left(\mu^{i}\right)_{o}$, we are to give to $\left(\lambda^{i}\right)_{o}$ values consistent with (165) and

$$
\left(a_{i j} \lambda^{i} \lambda^{j}\right)_{O}=1,
$$

but otherwise arbitrary. The critical region on $O P$ is confined between minimum and maximum values of $r$ as given by (184). Hence we may state the following result:

THEOREM XVI. When a system performs oscillations about a position of stable equilibrium, then, if the total energy $E$ is sufficiently small, there is one critical region with respect to potential-apsides in the region of motion $(V<E)$, and it lies between the surfaces whose polar equations are

$$
\begin{aligned}
& r=E^{1 / 2}\left(f_{0}^{\prime}+f_{1}^{\prime} E^{1 / 2}+f_{2}^{\prime} E+\cdots\right), \\
& r=E^{1 / 2}\left(f_{0}^{\prime \prime}+f_{1}^{\prime \prime} E^{1 / 2}+f_{2}^{\prime \prime} E+\cdots\right),
\end{aligned}
$$

where the single accent denotes minimum values, and the double accent maximum values, of certain quantities depending on the initial direction $\left(\mu^{i}\right)_{o}$ of the geodesic along which $r$ is measured; the minimum and maximum values are with respect to values of $\left(\lambda^{i}\right)_{0}$ which are arbitrary but for (165) and (187).

There is rather a remarkable difference with regard to critical regions for radial-apsides and potential-apsides for oscillations about a position of stable equilibrium. We have already remarked ( $\$ 9$, after Theorem VII) on the order of the thickness of the critical region for radial-apsides. The linear dimensions of the ellipsoid $V=E$ are of the order of $E^{1 / 2}$, and the thickness of the critical region is of the order of $E^{3 / 2}$, showing that as $E$ tends to zero, the volume of the critical region bears to the volume of the region $V<E$ a vanishing ratio, in fact, a ratio of the order of $E$. On the other hand, we see from (186) that ${ }_{2}$ except under special circumstances, $f_{0}^{\prime}$ and $f_{0}^{\prime \prime}$ will differ from one 
another. The portion of the radial geodesic lying in the critical region will be of the order of $E^{1 / 2}$, which is the order of the radius vector to $V=E$. Thus, as $E$ tends to zero, the volume of the critical region for potential-apsides will bear to the total volume of the region $V<E$ a limiting ratio which is greater than zero.

The "special circumstances" alluded to occur when the infinitesimal quadric

$$
\left(V_{i j}\right)_{o} \xi^{i} \xi^{j}=\text { const. }
$$

is a sphere. Then we have

$$
\left(V_{i j}\right)_{o}=h\left(a_{i j}\right)_{O} \quad(h=\text { const. }),
$$

and therefore

$$
f_{0}=h^{-1 / 2} \text {, }
$$

so that $f_{0}^{\prime}=f_{0}^{\prime \prime}$, and the thickness of the shell between the surfaces (188) and (189) is of the order of $E$. Thus, as $E$ tends to zero, the ratio of the volume of this shell to the total volume of the region $V<E$ tends to zero (as $E^{1 / 2}$ ).

UNIVERSITY OF TORONTO, Toronto, Canada 